\title{
MAXIMUM RECOVERABLE WORK, MINIMUM FREE ENERGY AND STATE SPACE IN LINEAR VISCOELASTICITY
}

\author{
BY \\ GIORGIO GENTILI ${ }^{\dagger}$
}

Dipartimento di Matematica, Università di Bologna, P.za di P.ta S. Donato 5, 40126 Bologna, Italy

\begin{abstract}
The various formulations of the maximum recoverable work used in the literature are proved to be equivalent. Then an explicit formula of the minimum free energy is derived, starting from the formulation of the maximum recoverable work given by Day. The resulting expression is equivalent to that found by Golden and other authors. However, the particular formulation allows us to prove that the domain of definition of minimum free energy is the whole state space. Finally, the maximum recoverable work is shown to be put as the basis of the thermodynamics of viscoelastic materials under isothermal conditions. In this context the usual relation between the Clausius-Duhem inequality and the dissipation of the material is restored.
\end{abstract}

1. Introduction. All the definitions of Helmholtz free energy for a viscoelastic material (for instance, the one given by Graffi $[11,18,19,20]$ and that stated by Coleman and Owen $[3,7,8]$ ) do not identify a unique functional. Moreover, it has been shown [23] that in the convex set of all free energies there exist maximum and minimum elements. An explicit form of the maximum free energy, according to Graffi's definition, has been given by Fabrizio, Giorgi and Morro in [11]. Instead the minimum free energy, according to both Graffi's and Coleman-Owen's definitions, has been proved to be obtained by maximizing the recoverable work.

For this reason, in the literature we can find many papers discussing the expression of the maximum recoverable work in linear viscoelasticity.

However, the procedure for finding such an expression, and hence an explicit form of the minimum free energy, is nothing but easy.

The most important work in this sense is due to Day $[4,5]$. Nevertheless, he gave an interesting characterization of the maximum recoverable work, but not an explicit

Received March 1, 2000.

2000 Mathematics Subject Classification. Primary 74A15, 74D99; Secondary 45E10.

This work has been done under the auspices of G.N.F.M. of I.N.D.A.M., partially supported by the Italian MURST through the COFIN'98 Research Program "Modelli Matematici per la Scienza dei Materiali" and partially supported by University of Bologna Funds for selected research topics.

The author wishes to thank professors M. Fabrizio, C. Giorgi and J. M. Golden for helpful discussions. ${ }^{\dagger}$ Deceased 3rd December, 2000. 
formula. Recently an expression for the maximum recoverable work, and the minimum free energy, was found by Golden [17] in the scalar case and then extended to the general isothermal case $[9,13]$.

However, some questions seem to be still open.

For instance, although the definition of maximum recoverable work is clear, some different formulations have been used to obtain the above quoted results. So, the question whether such formulations are equivalent arises.

Secondly, it is worth recalling that the space of definition of any free energy in general is a proper subset of the whole state space. In other words, a free energy may be unbounded in correspondence with some strain history. On the other hand, it is reasonable to think that the domain of the minimum free energy is wider than the one corresponding to any other free energy. As a consequence we should expect that such a space does coincide with the whole state space if properly defined.

In this paper we try to answer the above questions.

First of all, we give an alternative definition of the state space based on the boundedness of the work rather than the stress (Sec. 3).

Secondly, we give a rigorous proof that all the formulations of the maximum recoverable work present in the literature are equivalent (Sec. 4). What is more, this is proved avoiding any topology on the state space. In materials with memory and in particular in viscoelasticity, this is important because many different and unequivalent topologies may be given, but the objective characteristics of the material, as well as thermodynamics and its implications, must be unaffected by the particular choice of the topology.

Then (Sec. 5), thanks to previous results given in $[17,9]$, we are able to prove that the point of view of Day leads to an explicit expression of the minimum free energy that is perfectly equivalent to that found in [17] and [9] . Moreover (Sec. 6), such an expression allows us to prove that the domain of definition of the minimum free energy is the whole state space as defined in Sec. 3 .

Finally (Sec. 7), we show that the existence and the boundedness of the maximum recoverable work on the whole state space can be put as a basis of the thermodynamics of viscoelastic materials under isothermal conditions, as already drafted by Fabrizio, Giorgi and Morro. Such an approach allows us to formulate the dissipativity properties of the material without reference to any particular topology. Moreover, in describing the properties of the maximum recoverable work and identifying it with the minimum free energy, we are led to an interesting physical consideration that enables us to go deep into the connection between the Clausius-Duhem inequality and the dissipation of the material. In fact, some authors (see, e.g., [23]) claimed that they are unrelated when memory effects occur because of the nonuniqueness of the free energy functional. On the contrary, we prove that such a relation is restored whenever the free energy involved in the Clausius-Duhem inequality is the minimum one.

2. Notation and basic assumptions for a linear viscoelastic solid. Let Sym be the space of symmetric second-order tensors acting on $\mathcal{R}^{3}$ endowed with the inner product $\mathbf{A} \cdot \mathbf{B}:=\operatorname{tr}\left(\mathbf{A B}^{\top}\right)$ and norm $|\mathbf{A}|=(\mathbf{A} \cdot \mathbf{A})^{1 / 2}$ where "T" denotes the transpose. Also let $\operatorname{Lin}($ Sym $)$ denote the space of the fourth-order tensors operating on Sym. For 
any $\mathbb{K} \in \operatorname{Lin}(S y m)$, the transpose $\mathbb{K}^{\top}$ is such that $\mathbb{K}^{\top} \mathbf{A} \cdot \mathbf{B}=\mathbb{K} \mathbf{B} \cdot \mathbf{A}, \forall \mathbf{A}, \mathbf{B} \in S y m$. The reason for this notation is clear by recalling that $S y m$ is isomorphic to $\mathcal{R}^{6}$. Therefore, $\mathbf{C}_{i}, i=1, \ldots, 6$ is an orthonormal basis of $S y m$ so that

$$
\mathbf{A}=\sum_{i=1}^{6} A_{i} \mathbf{C}_{i}, \quad \mathbf{B}=\sum_{i=1}^{6} B_{i} \mathbf{C}_{i}, \quad \mathbf{A} \cdot \mathbf{B}=\sum_{i=1}^{6} A_{i} B_{i} .
$$

Analogously any tensor $\mathbb{K} \in \operatorname{Lin}(S y m)$ will be identified with an element of $\operatorname{Lin}\left(\mathcal{R}^{6}\right)$ by the representation

$$
\mathbb{K}=\sum_{i, i=1}^{6} K_{i j} \mathbf{C}_{i} \otimes \mathbf{C}_{j}
$$

and $\mathbb{K}^{\top}$ is the transpose of $\mathbb{K}$ as an element of $\operatorname{Lin}\left(\mathcal{R}^{6}\right)$. According to that, the norm $|\mathbb{K}|$ of $\mathbb{K} \in \operatorname{Lin}($ Sym $)$ may be given by

$$
|\mathbb{K}|^{2}=\operatorname{tr}\left(\mathbb{K} \mathbb{K}^{\top}\right)=\left(\sum_{i, j=1}^{6} K_{i j} K_{j i}\right) .
$$

In the sequel we deal with complex-valued tensors. Denoting by $\Omega$ the complex plane and by $\operatorname{Sym}(\Omega)$ and $\operatorname{Lin}(\operatorname{Sym}(\Omega))$ respectively the tensors represented by the above forms with $A_{i}, B_{i}, K_{i j} \in \Omega$, then the norms $|\mathbf{A}|$ and $|\mathbb{K}|$ of $\mathbf{A} \in \operatorname{Sym}(\Omega)$ and $\mathbb{K} \in \operatorname{Lin}(\operatorname{Sym}(\Omega))$ will be given respectively by

$$
|\mathbf{A}|^{2}=(\mathbf{A} \cdot \overline{\mathbf{A}}), \quad|\mathbb{K}|^{2}=\operatorname{tr}\left(\mathbb{K} \mathbb{K}^{*}\right)=\left(\sum_{i, j=1}^{6} K_{i j} \bar{K}_{j i}\right),
$$

where the overhead bar indicates complex conjugate and $\mathbb{K}^{*}=\overline{\mathbb{K}}^{\top}$. The above representations allow all results of [16] to be easily extended to tensors belonging to $\operatorname{Lin}(\operatorname{Sym}(\Omega))$.

For any function $f \in L^{1}(\mathcal{R}, V) \cup L^{2}(\mathcal{R}, V)$, where $V$ is a finite-dimensional vector space (in the present context $S y m$ or $\operatorname{Lin}(S y m)$ ), let $f_{F}$ denote its Fourier transform viz. $f_{F}(\omega)=\int_{-\infty}^{\infty} f(s) e^{-i \omega s} d s$. Also, we define

$$
\begin{aligned}
& f_{+}(\omega)=\int_{0}^{\infty} f(s) e^{-i \omega s} d s, \quad f_{-}(\omega)=\int_{-\infty}^{0} f(s) e^{-i \omega s} d s \\
& f_{s}(\omega)=\int_{0}^{\infty} f(s) \sin \omega s d s, \quad f_{c}(\omega)=\int_{0}^{\infty} f(s) \cos \omega s d s .
\end{aligned}
$$

All these relations are to be understood as applying to each component of the tensor quantities involved.

In the sequel, we need to consider the Fourier transform of functions that do not vanish at large times and thus do not belong to $L^{2}$ for the appropriate domain. The standard procedure is adopted of introducing an exponential decay factor, calculating the Fourier transform and letting the time decay constant tend to infinity. Thus, if $f$ is a function defined on $\mathcal{R}^{+}$such that $\lim _{s \rightarrow+\infty} f(s)=f_{\infty}$, and $g: \mathcal{R}^{+} \rightarrow \mathcal{V}$, defined by $g(s)=f(s)-f_{\infty}$, belongs to $L^{1}\left(\mathcal{R}^{+}, \mathcal{V}\right) \cap L^{2}\left(\mathcal{R}^{+}, \mathcal{V}\right)$, then

$$
f_{F}(\omega)=g_{F}(\omega)-\frac{f_{\infty}}{i \omega^{+}}, \quad \omega^{+}=\lim _{\alpha \rightarrow 0^{+}}(\omega+i \alpha) .
$$


Henceforth $\Omega^{+}$and $\Omega^{(+)}$denote the following sets:

$$
\Omega^{+}=\left\{\zeta \in \Omega: \Im_{m} \zeta \geq 0\right\}, \quad \Omega^{(+)}=\left\{\zeta \in \Omega: \Im_{m} \zeta>0\right\}
$$

and analogous meaning is stated for $\Omega^{-}$and $\Omega^{(-)}$. The quantities $f_{ \pm}(z)$ defined in $(2.1)$ are analytic for $z \in \Omega^{(\mp)}$.

A linear viscoelastic material is described by the classical Boltzmann-Volterra constitutive equation between the stress tensor $\mathbf{T}(t) \in S y m$ and the strain history tensor $\mathbf{E}:(-\infty, t] \rightarrow S y m$ of the form:

$$
\mathbf{T}(t)=\mathbb{G}_{0} \mathbf{E}(t)+\int_{0}^{\infty} \dot{\mathbb{G}}(s) \mathbf{E}^{t}(s) d s,
$$

where $\mathbf{E}(t) \in S y m$ is the instantaneous value of the strain and $\mathbf{E}^{t}:(0,+\infty) \rightarrow$ Sym denotes the past history defined as

$$
\mathbf{E}^{t}(s):=\mathbf{E}(t-s), \quad s \in \mathcal{R}^{++}:=(0,+\infty) .
$$

Henceforth for simplicity we identify the strain history with the couple $\left(\mathbf{E}(t), \mathbf{E}^{t}\right)$ and use the term "history" just to indicate the past history $\mathbf{E}^{t}$. Since $\dot{\mathbb{G}} \in L^{1}\left(\mathcal{R}^{+}, \operatorname{Lin}(\operatorname{Sym})\right)$, its primitive, the relaxation function $\mathbb{G}:(0,+\infty) \rightarrow \operatorname{Lin}(\operatorname{Sym})$ such that

$$
\mathbb{G}(t)=\mathbb{G}_{0}+\int_{0}^{t} \dot{\mathbb{G}}(s) d s
$$

is well defined. The quantity $\mathbb{G}_{0}=\mathbb{G}(0)$ is named as the instantaneous elastic modulus. Moreover, there exists the limit

$$
\mathbb{G}_{\infty}:=\lim _{t \rightarrow \infty} \mathbb{G}(t) \in \operatorname{Lin}(\text { Sym }),
$$

where $\mathbb{G}_{\infty}$ is named as the equilibrium elastic modulus.

We also assume that $\check{G}:(0,+\infty) \rightarrow \operatorname{Lin}(\operatorname{Sym})$, defined as

$$
\check{\mathbb{G}}(t)=\mathbb{G}(t)-\mathbb{G}_{\infty},
$$

is symmetric, integrable and its integral does not vanish, viz.

$$
\check{\mathbb{G}}(t)=\check{\mathbb{G}}^{\top}(t), \quad 0<\left|\int_{0}^{\infty} \check{\mathbb{G}}(s) d s\right|<\infty .
$$

The thermodynamic properties of a linear viscoelastic material imply (see $[15,14]$ )

$$
\mathbb{G}_{\infty}=\mathbb{G}_{\infty}^{\top}, \quad \dot{\mathbb{G}}_{s}(\omega) \mathbf{E} \cdot \mathbf{E}<0 \quad \forall \mathbf{E} \in \operatorname{Sym} \backslash\{\mathbf{0}\} \forall \omega \in(0,+\infty) .
$$

Note that $(2.5)_{2}$ may be written in terms of the Fourier transform of $\check{G}$ by virtue of the identity

$$
\dot{\mathbb{G}}_{s}(\omega)=-\omega \check{\mathbb{G}}_{c}(\omega)
$$

Moreover, $(2.4)_{2}$ and $(2.5)_{2}$ imply

$$
\check{\mathbb{G}}_{c}(\omega) \mathbf{E} \cdot \mathbf{E}>0 \quad \forall \mathbf{E} \in S y m \backslash\{\mathbf{0}\} \forall \omega \in \mathcal{R} .
$$

Moreover, on the basis of physical arguments, the equilibrium elastic modulus of a viscoelastic solid is assumed to be positive definite, namely

$$
\mathbb{G}_{\infty} \mathbf{E} \cdot \mathbf{E}>0, \quad \forall \mathbf{E} \in S y m \backslash\{\mathbf{0}\} .
$$


Further, we assume

$$
\dot{\mathbb{G}}(0) \mathbf{E} \cdot \mathbf{E}<0, \quad \forall \mathbf{E} \in S y m \backslash\{\mathbf{0}\},
$$

whereas inequality $(2.5)_{2}$ just implies that $\dot{\mathbb{G}}(0)$ is negative semidefinite (see [15]).

If we define the vectorial space

$$
\Gamma:=\left\{\mathbf{E}^{t}:(0,+\infty) \rightarrow \text { Sym } ;\left|\int_{0}^{\infty} \dot{\mathbb{G}}(s+\tau) \mathbf{E}^{t}(s) d s\right|<\infty \quad \forall \tau \geq 0\right\},
$$

whose definition depends on the relaxation function, the Boltzman-Volterra equation (2.2) defines the linear functional $\tilde{\mathbf{T}}: S y m \times \Gamma \rightarrow$ Sym such that

$$
\tilde{\mathbf{T}}\left(\mathbf{E}(t), \mathbf{E}^{t}\right)=\mathbb{G}_{0} \mathbf{E}(t)+\int_{0}^{\infty} \dot{\mathbb{G}}(s) \mathbf{E}^{t}(s) d s .
$$

Given the strain $\left(\mathbf{E}(t), \mathbf{E}^{t}\right)$ continued with $\mathbf{E}(t+a)=\mathbf{E}(t), a \in \mathcal{R}^{+}$, it is easy to check that the yielded stress is given by

$$
\mathbf{T}(t+a)=\mathbb{G}(a) \mathbf{E}(t)+\int_{0}^{\infty} \dot{\mathbb{G}}(s+a) \mathbf{E}^{t}(s) d s .
$$

It has been shown (see [9], Proposition 2.2, (ii)), that the fading memory property $\dot{\mathbb{G}} \in L^{1}$ ensures that for every $\varepsilon>0$ there exists $a\left(\varepsilon, \mathbf{E}^{t}\right)$ sufficiently large such that

$$
\left|\int_{0}^{\infty} \dot{\mathbb{G}}(s+a) \mathbf{E}^{t}(s) d s\right|<\varepsilon, \quad \forall a>a\left(\varepsilon, \mathbf{E}^{t}\right) .
$$

\section{A new definition of the space of the processes and of the state space.} The notions of the state and the process for a linear viscoelastic solid have been discussed by various authors (see $[10,8,21,24])$. They can be resumed as follows.

REMARK 3.1. According to the definition given in [6] and [15], a process $P$ of duration $d<+\infty$ is given by $\dot{\mathbf{E}}_{P}:[0, d) \rightarrow S y m$. For simplicity, given the $\operatorname{strain}\left(\mathbf{E}(t), \mathbf{E}^{t}\right) \in$ Sym $\times \Gamma$, we relate $P$ to

$$
\mathbf{E}_{P}:[0, d) \rightarrow \text { Sym }, \quad \mathbf{E}_{P}(\tau)=\mathbf{E}(t)+\int_{0}^{\tau} \dot{\mathbf{E}}_{P}\left(s^{\prime}\right) d s^{\prime}, \quad \tau \in(0, d]
$$

Thus the strain $\mathbf{E}_{f}\left(\tau^{\prime}\right)=\left(\mathbf{E}_{P} * \mathbf{E}\right)\left(\tau^{\prime}\right), \tau^{\prime} \leq t+d$, yielded by $\mathbf{E}^{t}$ and $\dot{\mathbf{E}}_{P}$, is related to the couple $\left(\mathbf{E}_{P}(d),\left(\mathbf{E}_{P} * \mathbf{E}\right)^{t+d}\right)$ and is given by

$$
\mathbf{E}_{f}(t+d-s)=\left(\mathbf{E}_{P} * \mathbf{E}\right)(t+d-s):= \begin{cases}\mathbf{E}_{P}(d-s) & \text { for } 0 \leq s<d, \\ \mathbf{E}(t+d-s) & \text { for } s \geq d\end{cases}
$$

In the sequel we use the symbol "*" to denote both the continuation of histories and the continuation of processes (see [8]).

Definition 3.2. (see [10]) Two strain histories $\left(\mathbf{E}_{1}, \mathbf{E}_{1}^{t}\right)$ and $\left(\mathbf{E}_{2}, \mathbf{E}_{2}^{t}\right)$ are said to be equivalent if for every $\mathbf{E}_{P}:(0, \tau) \rightarrow$ Sym and for every $\tau>0$, they satisfy

$$
\tilde{\mathbf{T}}\left(\mathbf{E}_{P}(\tau),\left(\mathbf{E}_{P} * \mathbf{E}_{1}\right)^{t+\tau}\right)=\tilde{\mathbf{T}}\left(\mathbf{E}_{P}(\tau),\left(\mathbf{E}_{P} * \mathbf{E}_{2}\right)^{t+\tau}\right) .
$$


As a consequence, it is easy to show that $\left(\mathbf{0}, \mathbf{E}^{t}\right)$ is equivalent to the zero history $\left(\mathbf{0}, \mathbf{0}^{\dagger}\right)$ if

$$
\int_{\tau}^{\infty} \dot{\mathbb{G}}(s) \mathbf{E}^{t+\tau}(s) d s=\int_{0}^{\infty} \dot{\mathbb{G}}(s+\tau) \mathbf{E}^{t}(s) d s=0 \quad \forall \tau>0 .
$$

Equality (3.4) represents an equivalence relation, i.e., two past histories $\mathbf{E}_{1}^{t}$ and $\mathbf{E}_{2}^{t}$ are said to be equivalent if their difference $\mathbf{E}^{t}=\mathbf{E}_{1}^{t}-\mathbf{E}_{2}^{t}$ satisfies (3.4).

According to the definition of state $\sigma$ given by Noll [24], two couples $\left(\mathbf{E}_{1}(t), \mathbf{E}_{1}^{t}\right)$ and $\left(\mathbf{E}_{2}(t), \mathbf{E}_{2}^{t}\right)$ such that $\mathbf{E}_{1}(t)=\mathbf{E}_{2}(t)$ and $\mathbf{E}_{1}^{t}-\mathbf{E}_{2}^{t}$ satisfies (3.4) are represented by the same state $\sigma(t)$. In this sense $\sigma(t)$ may be thought as the "minimum" set of variables allowing a univocal relation between $\dot{\mathbf{E}}_{P}:(0, \tau) \rightarrow$ Sym and the stress $\mathbf{T}(t+\tau)=$ $\tilde{\mathbf{T}}\left(\mathbf{E}_{P}(\tau),\left(\mathbf{E}_{P} * \mathbf{E}\right)^{t+\tau}\right)$ for every $\tau>0$.

In other words (see $[8,21]$ ), denoting by $\Gamma_{0}$ the set of all the past histories of $\Gamma$ satisfying (3.4), and by $\Gamma / \Gamma_{0}$ the usual quotient space, the state $\sigma$ of a linear viscoelastic material may be identified by a couple $(\mathbf{E}, \mathbf{h})$ with $\mathbf{E} \in S y m$ and $\mathbf{h} \in \Gamma / \Gamma_{0}$ and the state space may be identified as

$$
\Sigma=\operatorname{Sym} \times\left(\Gamma / \Gamma_{0}\right) .
$$

In this paper we present a different point of view in defining the state space. Roughly speaking, if $\Sigma=S y m \times\left(\Gamma / \Gamma_{0}\right)$ is related to the boundedness of the stress (see, e.g., $(2.10)$ ), we now construct a new state space related to the boundedness of the work. Such a procedure also induces a suitable definition of the space of the processes.

Given the initial strain described by the pair $\left(\mathbf{E}_{i}(t), \mathbf{E}_{i}^{t}\right)$ and the process $P$ given by $\dot{\mathbf{E}}_{P}:(0, d] \rightarrow S y m$, the work done on $P$, is denoted by $\widetilde{W}\left(\mathbf{E}_{i}(t), \mathbf{E}_{i}^{t} ; \dot{\mathbf{E}}_{P}\right)$, viz.

$$
\begin{aligned}
\widetilde{W}\left(\mathbf{E}_{i}(t), \mathbf{E}_{i}^{t} ; \dot{\mathbf{E}}_{P}\right):= & \int_{0}^{d} \tilde{\mathbf{T}}\left(\mathbf{E}_{P}(\tau),\left(\mathbf{E}_{P} * \mathbf{E}_{i}\right)^{t+\tau}\right) \cdot \dot{\mathbf{E}}_{P}(\tau) d \tau \\
= & \int_{t}^{t+d} \tilde{\mathbf{T}}\left(\mathbf{E}(\tau), \mathbf{E}^{\tau}\right) \cdot \dot{\mathbf{E}}(\tau) d \tau \\
= & \frac{1}{2} \mathbb{G}(0) \mathbf{E}(t+d) \cdot \mathbf{E}(t+d)-\frac{1}{2} \mathbb{G}(0) \mathbf{E}(t) \cdot \mathbf{E}(t) \\
& +\int_{t}^{t+d} \int_{0}^{\infty} \dot{\mathbb{G}}(s) \mathbf{E}^{\tau}(s) \cdot \dot{\mathbf{E}}(\tau) d \tau
\end{aligned}
$$

where $\mathbf{E}=\mathbf{E}_{P} * \mathbf{E}_{i}$ as defined in (3.2).

Given a process $\dot{\mathbf{E}}_{P}:[0, d) \rightarrow$ Sym and the null strain history $\left(\mathbf{0}, \mathbf{0}^{\dagger}\right)$, where $\mathbf{0}^{\dagger}(s)=\mathbf{0}$, $\forall s>0$, let $\left(\mathbf{E}_{0}(t), \mathbf{E}_{0}^{t}\right)$ denote the ensuing strain given by (for simplicity we now take the initial instant as $t=0$ )

$$
\mathbf{E}_{0}(t):=\int_{0}^{t} \dot{\mathbf{E}}_{P}(\tau) d \tau, \quad \mathbf{E}_{0}^{t}(s):= \begin{cases}\int_{0}^{t-s} \dot{\mathbf{E}}_{P}(\tau) d \tau & \text { for } 0<s \leq t \\ \mathbf{0} & \text { for } s>t\end{cases}
$$

Definition 3.3. A process $\dot{\mathbf{E}}_{P}:[0, d) \rightarrow S y m(d \leq \infty)$ is said to be a finite work process if

$$
\widetilde{W}\left(\mathbf{0}, \mathbf{0}^{\dagger} ; \dot{\mathbf{E}}_{P}\right)=\int_{0}^{d} \tilde{\mathbf{T}}\left(\mathbf{E}_{0}(t), \mathbf{E}_{0}^{t}\right) \cdot \dot{\mathbf{E}}_{P}(t) d t<\infty,
$$


where $\left(\mathbf{E}_{0}(t), \mathbf{E}_{0}^{t}\right)$ is defined by (3.8).

Observe that $\widetilde{W}\left(\mathbf{0}, \mathbf{0}^{\dagger} ; \dot{\mathbf{E}}_{P}\right)>0$ by virtue of the Dissipation Principle stated by Gurtin and Herrera (see [22]); however, it can also be derived as a consequence of $(2.5)_{2}$ and (2.8).

When $d<\infty$ we can consider $\dot{\mathbf{E}}_{P}: \mathcal{R}^{+} \rightarrow$ Sym with $\dot{\mathbf{E}}_{P}(t)=0$ for $t>d$ so that

$$
\begin{aligned}
\widetilde{W}\left(\mathbf{0}, \mathbf{0}^{\dagger} ; \dot{\mathbf{E}}_{P}\right) & =\int_{0}^{\infty}\left[\mathbb{G}_{0} \mathbf{E}_{0}(\tau)+\int_{0}^{\tau} \dot{\mathbb{G}}(s) \mathbf{E}_{0}(\tau-s) d s\right] \cdot \dot{\mathbf{E}}_{P}(\tau) d \tau \\
& =\int_{0}^{\infty} \int_{0}^{\tau} \mathbb{G}(\tau-s) \dot{\mathbf{E}}_{P}(s) \cdot \dot{\mathbf{E}}_{P}(\tau) d s d \tau \\
& =\frac{1}{2} \int_{0}^{\infty} \int_{0}^{\infty} \mathbb{G}(|\tau-s|) \dot{\mathbf{E}}_{P}(s) \cdot \dot{\mathbf{E}}_{P}(\tau) d s d \tau,
\end{aligned}
$$

where $\mathbb{G}(t)=\mathbb{G}_{0}+\int_{0}^{t} \dot{\mathbb{G}}(s) d s$. Using the function $\check{\mathbb{G}} \in L^{1}\left(\mathcal{R}^{+}, \operatorname{Lin}(\operatorname{Sym})\right)$ such that $\check{\mathbb{G}}(t)=\mathbb{G}(t)-\mathbb{G}_{\infty}$, we have

$$
\begin{aligned}
\widetilde{W}\left(\mathbf{0}, \mathbf{0}^{\dagger} ; \dot{\mathbf{E}}_{P}\right) & =\frac{1}{2} \mathbb{G}_{\infty} \mathbf{E}_{0}(d) \cdot \mathbf{E}_{0}(d)+\frac{1}{2} \int_{0}^{\infty} \int_{0}^{\infty} \check{\mathbb{G}}(|\tau-s|) \dot{\mathbf{E}}_{P}(s) \cdot \dot{\mathbf{E}}_{P}(\tau) d s d \tau \\
& =\frac{1}{2} \mathbb{G}_{\infty} \mathbf{E}_{0}(d) \cdot \mathbf{E}_{0}(d)+\frac{1}{2 \pi} \int_{-\infty}^{\infty} \check{\mathbb{G}}_{c}(\omega) \dot{\mathbf{E}}_{P+}(\omega) \cdot \dot{\mathbf{E}}_{P+}(\omega) d \omega .
\end{aligned}
$$

Therefore, the set of the finite work processes can be characterised by the following set:

$$
\check{\mathcal{H}}_{G}\left(\mathcal{R}^{+}, \text {Sym }\right):=\left\{\boldsymbol{\varphi}: \mathcal{R}^{+} \rightarrow \text { Sym }: \int_{-\infty}^{\infty} \check{\mathbb{G}}_{c}(\omega) \boldsymbol{\varphi}_{+}(\omega) \cdot \overline{\boldsymbol{\varphi}_{+}(\omega)} d \omega<\infty\right\} .
$$

Observe that, since $\check{\mathbb{G}}_{c}(\omega)$ is positive definite $\forall \omega \in \mathcal{R}, \check{\mathcal{H}}_{G}\left(\mathcal{R}^{+}, S y m\right)$ may be endowed with the inner product $(\cdot, \cdot)$ defined as

$$
\left(\boldsymbol{\varphi}_{1}, \boldsymbol{\varphi}_{2}\right)_{G}=\int_{-\infty}^{\infty} \check{\mathbb{G}}_{c}(\omega) \boldsymbol{\varphi}_{1+}(\omega) \cdot \overline{\boldsymbol{\varphi}_{2+}(\omega)} d \omega
$$

and the norm $\|\cdot\|_{G}$ such that $\|\varphi\|_{G}^{2}=(\varphi, \varphi)_{G}$. Therefore, we can define the space of the processes as the Hilbert space $\mathcal{H}_{G}\left(\mathcal{R}^{+}\right.$, Sym) obtained by the completion of $\check{\mathcal{H}}_{G}\left(\mathcal{R}^{+}\right.$, Sym $)$with respect to the norm $\|\cdot\|_{G}$.

Definition 3.4. The set of admissible strain histories is defined as the set of all the couples $\left(\mathbf{E}(t), \mathbf{E}^{t}\right)$ such that the work $\widetilde{W}\left(\mathbf{E}(t), \mathbf{E}^{t} ; \dot{\mathbf{E}}_{P}\right)$ is finite for every $\dot{\mathbf{E}}_{P} \in$ $\mathcal{H}_{G}\left(\mathcal{R}^{+}\right.$, Sym $)$.

Thus, the domain of definition of the stress is the set of all those strain histories rendering the work well defined when the process belongs to $\mathcal{H}_{G}\left(\mathcal{R}^{+}, S y m\right)$.

Observe that

$$
\begin{aligned}
& \widetilde{W}\left(\mathbf{E}(t), \mathbf{E}^{t} ; \dot{\mathbf{E}}_{P}\right) \\
& =\int_{0}^{\infty}\left[\mathbb{G}_{0} \mathbf{E}(t+\tau)+\int_{0}^{\tau} \dot{\mathbb{G}}(s) \mathbf{E}_{P}(\tau-s) d s+\int_{\tau}^{\infty} \dot{\mathbb{G}}(s) \mathbf{E}(t+\tau-s) d s\right] \cdot \dot{\mathbf{E}}_{P}(\tau) d \tau \\
& =\int_{0}^{\infty}\left[\mathbb{G}(\tau) \mathbf{E}(t)+\int_{0}^{\tau} \mathbb{G}(\tau-s) \dot{\mathbf{E}}_{P}(s) d s+\int_{0}^{\infty} \dot{\mathbb{G}}(\tau+s) \mathbf{E}^{t}(s) d s\right] \cdot \dot{\mathbf{E}}_{P}(\tau) d \tau \\
& =\int_{0}^{\infty}\left[\frac{1}{2} \int_{0}^{\infty} \mathbb{G}(|\tau-s|) \dot{\mathbf{E}}_{P}(s) d s-\mathbf{I}\left(\tau, \mathbf{E}(t), \mathbf{E}^{t}\right)\right] \cdot \dot{\mathbf{E}}_{P}(\tau) d \tau
\end{aligned}
$$


where

$$
\mathbf{I}\left(\tau, \mathbf{E}(t), \mathbf{E}^{t}\right)=-\mathbb{G}(\tau) \mathbf{E}(t)-\int_{0}^{\infty} \dot{\mathbb{G}}(\tau+s) \mathbf{E}^{t}(s) d s, \quad \tau \geq 0 .
$$

Let $\breve{\mathbf{E}}^{t}(s)=\mathbf{E}^{t}(s)-\mathbf{E}(t)$ be the history relative to the instantaneous value. It is obvious that a strain history can be described equivalently by the couple $\left(\mathbf{E}(t), \mathbf{E}^{t}\right)$ or by $\left(\mathbf{E}(t), \breve{\mathbf{E}}^{t}\right)$. Let $\breve{\mathbf{I}}\left(\cdot, \breve{\mathbf{E}}^{t}\right): \mathcal{R}^{+} \rightarrow$ Sym be defined by

$$
\breve{\mathbf{I}}\left(\tau, \breve{\mathbf{E}}^{t}\right)=-\int_{0}^{\infty} \dot{\mathbb{G}}(\tau+s) \breve{\mathbf{E}}^{t}(s) d s, \quad \tau \geq 0 ;
$$

we have

$$
\mathbf{I}\left(\tau, \mathbf{E}(t), \mathbf{E}^{t}\right)=-\mathbb{G}_{\infty} \mathbf{E}(t)+\breve{\mathbf{I}}\left(\tau, \breve{\mathbf{E}}^{t}\right), \quad \tau \geq 0
$$

and

$$
\begin{aligned}
\widetilde{W}\left(\mathbf{E}(t), \mathbf{E}^{t} ; \dot{\mathbf{E}}_{P}\right)= & \frac{1}{2} \mathbb{G}_{\infty} \mathbf{E}_{0}(d) \cdot \mathbf{E}_{0}(d)+\frac{1}{2} \int_{0}^{\infty} \int_{0}^{\infty} \check{\mathbb{G}}(|\tau-s|) \dot{\mathbf{E}}_{P}(s) \cdot \dot{\mathbf{E}}_{P}(\tau) d s d \tau \\
& +\mathbb{G}_{\infty} \mathbf{E}(t) \cdot \mathbf{E}_{0}(d)-\int_{0}^{\infty} \breve{\mathbf{I}}\left(\tau, \breve{\mathbf{E}}^{t}\right) \cdot \dot{\mathbf{E}}_{P}(\tau) d \tau
\end{aligned}
$$

where $\mathbf{E}_{0}$ is given by $(3.8)_{1}$. Expression (3.16) shows how the work may be written in terms of the couple $\left(\mathbf{E}(t), \breve{\mathbf{E}}^{t}\right)$ and the process $\dot{\mathbf{E}}_{P}$. We denote $\widehat{W}\left(\mathbf{E}(t), \breve{\mathbf{E}}^{t} ; \dot{\mathbf{E}}_{P}\right)=$ $\widetilde{W}\left(\mathbf{E}(t), \mathbf{E}^{t} ; \dot{\mathbf{E}}_{P}\right)$. Moreover, by means of Plancherel's theorem we have

$$
\begin{aligned}
\widehat{W}\left(\mathbf{E}(t), \breve{\mathbf{E}}^{t} ; \dot{\mathbf{E}}_{P}\right)= & \frac{1}{2} \mathbb{G}_{\infty} \mathbf{E}_{0}(d) \cdot \mathbf{E}_{0}(d)+\frac{1}{2 \pi} \int_{-\infty}^{\infty} \check{\mathbb{G}}_{c}(\omega) \dot{\mathbf{E}}_{P+}(\omega) \cdot \overline{\dot{\mathbf{E}}_{P+}(\omega)} d \omega \\
& +\mathbb{G}_{\infty} \mathbf{E}(t) \cdot \mathbf{E}_{0}(d)-\frac{1}{2 \pi} \int_{-\infty}^{\infty} \breve{\mathbf{I}}_{+}\left(\omega, \breve{\mathbf{E}}^{t}\right) \cdot \overline{\dot{\mathbf{E}}_{P+}(\omega)} d \omega,
\end{aligned}
$$

where $\breve{\mathbf{I}}_{+}\left(\omega, \breve{\mathbf{E}}^{t}\right)=\int_{0}^{\infty} \breve{\mathbf{I}}\left(\tau, \breve{\mathbf{E}}^{t}\right) e^{-i \omega \tau} d \tau$ and

$$
\langle\mathbf{f}, \boldsymbol{\varphi}\rangle=\int_{0}^{\infty} \mathbf{f}(t) \cdot \boldsymbol{\varphi}(t) d t=\frac{1}{2 \pi} \int_{-\infty}^{\infty} \mathbf{f}_{+}(\omega) \cdot \overline{\boldsymbol{\varphi}_{+}(\omega)} d \omega,
$$

provided the integral is finite.

Therefore, the set of admissible strain histories can be thought of as the set of all the couples $\left(\mathbf{E}(t), \breve{\mathbf{E}}^{t}\right)$ such that $\mathbf{E}(t) \in S y m$ and the quantity $\breve{\mathbf{I}}\left(\cdot, \mathbf{E}^{t}\right)$, related to $\breve{\mathbf{E}}^{t}$ by (3.14), belongs to the dual of $\mathcal{H}_{G}\left(\mathcal{R}^{+}, S y m\right)$, namely to

$$
\mathcal{H}_{G}^{\prime}\left(\mathcal{R}^{+}, \text {Sym }\right):=\left\{\mathbf{f}: \mathcal{R}^{+} \rightarrow \text { Sym }:|\langle\mathbf{f}, \boldsymbol{\varphi}\rangle|<\infty, \quad \forall \boldsymbol{\varphi} \in \mathcal{H}_{G}\left(\mathcal{R}^{+}, \text {Sym }\right)\right\} .
$$

REMARK 3.5. The quantity $\breve{\mathbf{I}}\left(\tau, \breve{\mathbf{E}}^{t}\right)$ is related to the stress associated to the static continuation of $\mathbf{E}^{t}$ defined as

$$
\mathbf{E}_{c}^{t+\tau}(s)=\left\{\begin{array}{ll}
\mathbf{E}(t) & \text { for } s \leq \tau, \\
\mathbf{E}^{t}(s-\tau) & \text { for } s>\tau,
\end{array} \quad \tau>0\right.
$$

In fact in this case, we have

$$
\tilde{\mathbf{T}}\left(\mathbf{E}_{c}^{t+\tau}\right)=\mathbb{G}(\tau) \mathbf{E}(t)+\int_{0}^{\infty} \dot{\mathbb{G}}(\tau+s) \mathbf{E}^{t}(s) d s=\mathbb{G}_{\infty} \mathbf{E}(t)-\breve{\mathbf{I}}\left(\tau, \breve{\mathbf{E}}^{t}\right) .
$$


Therefore, the requested regularity on $\breve{\mathbf{I}}\left(\tau, \breve{\mathbf{E}}^{t}\right)$ is the regularity of

$$
\breve{\mathbf{T}}\left(\mathbf{E}_{c}^{t+\tau}\right)=\tilde{\mathbf{T}}\left(\mathbf{E}_{c}^{t+\tau}\right)-\mathbb{G}_{\infty} \mathbf{E}(t) .
$$

In particular, the fading memory property implies that $\lim _{\tau \rightarrow \infty} \breve{\mathbf{T}}\left(\mathbf{E}_{c}^{t+\tau}\right)=\mathbf{0}$.

In the previous section we called as equivalent two strain histories $\left(\mathbf{E}_{1}(t), \mathbf{E}_{1}^{t}\right)$ and $\left(\mathbf{E}_{2}(t), \mathbf{E}_{2}^{t}\right)$ that yield the same stress when subjected to the same process. The analogous equivalence relation may be done by means of the work.

Definition 3.6. Two strain histories $\left(\mathbf{E}_{1}(t), \breve{\mathbf{E}}_{1}^{t}\right)$ and $\left(\mathbf{E}_{2}(t), \breve{\mathbf{E}}_{2}^{t}\right)$ are said to be $w$ equivalent if for every $\dot{\mathbf{E}}_{P}:(0, \tau) \rightarrow$ Sym and for every $\tau>0$, they satisfy

$$
\widehat{W}\left(\mathbf{E}_{1}(t), \breve{\mathbf{E}}_{1}^{t} ; \dot{\mathbf{E}}_{P}\right)=\widehat{W}\left(\mathbf{E}_{2}(t), \breve{\mathbf{E}}_{2}^{t} ; \dot{\mathbf{E}}_{P}\right) .
$$

It is easy to check the following proposition.

Proposition 3.7. For every viscoelastic material described by the constitutive equation (2.2), two strain histories are $w$-equivalent if and only if they are equivalent in the sense of Definition 3.2 .

Proof. It is obvious that if $\left(\mathbf{E}_{1}(t), \mathbf{E}_{1}^{t}\right)$ and $\left(\mathbf{E}_{2}(t), \mathbf{E}_{2}^{t}\right)$ satisfy Definition 3.2 , then they are $w$-equivalent, namely $\left(\mathbf{E}_{1}(t), \breve{\mathbf{E}}_{1}^{t}\right)$ and $\left(\mathbf{E}_{2}(t), \breve{\mathbf{E}}_{2}^{t}\right)$ satisfy (3.18) for every $\mathbf{E}_{P}$ : $(0, d) \rightarrow$ Sym and for every $d>0$, since we have

$$
\int_{0}^{d} \tilde{\mathbf{T}}\left(\mathbf{E}_{P 1}(\tau),\left(\mathbf{E}_{P 1} * \mathbf{E}_{1}\right)^{t+\tau}\right) \cdot \dot{\mathbf{E}}_{P}(\tau) d \tau=\int_{0}^{d} \tilde{\mathbf{T}}\left(\mathbf{E}_{P 2}(\tau),\left(\mathbf{E}_{P 2} * \mathbf{E}_{1}\right)^{t+\tau}\right) \cdot \dot{\mathbf{E}}_{P}(\tau) d \tau
$$

the left [right] hand side being the definition of the work done by $\dot{\mathbf{E}}_{P}$ starting from $\left(\mathbf{E}_{1}(t), \mathbf{E}_{1}^{t}\right)\left[\left(\mathbf{E}_{2}(t), \mathbf{E}_{2}^{t}\right)\right]$. On the other hand, by virtue of $(3.16)$ and of the arbitrariness of $\dot{\mathbf{E}}_{P}$ and $d$, if $\left(\mathbf{E}_{1}(t), \breve{\mathbf{E}}_{1}^{t}\right)$ and $\left(\mathbf{E}_{2}(t), \breve{\mathbf{E}}_{2}^{t}\right)$ are $w$-equivalent, then they satisfy

$$
\mathbf{E}_{1}(t)=\mathbf{E}_{2}(t), \quad \mathbf{I}\left(\tau, \breve{\mathbf{E}}_{1}^{t}\right)=\mathbf{I}\left(\tau, \breve{\mathbf{E}}_{2}^{t}\right) \quad \forall \tau>0 .
$$

By virtue of (3.13) and (3.15), equalities (3.19) imply

$$
\int_{0}^{\infty} \dot{\mathbb{G}}(\tau+s) \mathbf{E}_{1}^{t}(s) d s=\int_{0}^{\infty} \dot{\mathbb{G}}(\tau+s) \mathbf{E}_{2}^{t}(s) d s \quad \forall \tau>0,
$$

namely, the difference $\mathbf{E}^{t}=\mathbf{E}_{1}^{t}-\mathbf{E}_{2}^{t}$ satisfies (3.4).

Therefore, if $\Gamma_{w}$ and $\Gamma_{w 0}$ denote respectively

$$
\begin{aligned}
\Gamma_{w} & =\left\{\breve{\mathbf{E}}^{t}: \mathcal{R}^{+} \rightarrow \text { Sym }: \breve{\mathbf{I}}\left(\cdot, \breve{\mathbf{E}}^{t}\right) \in \mathcal{H}_{G}^{\prime}\left(\mathcal{R}^{+}, \text {Sym }\right)\right\}, \\
\Gamma_{w 0} & =\left\{\breve{\mathbf{E}}^{t} \in \Gamma_{w}: \breve{\mathbf{I}}\left(\tau, \breve{\mathbf{E}}^{t}\right)=0, \quad \forall \tau>0\right\}
\end{aligned}
$$

then the new state space may be defined as

$$
\Sigma_{w}=\operatorname{Sym} \times\left(\Gamma_{w} / \Gamma_{w 0}\right) .
$$

As a consequence of Definition 3.2, the work is a function of the state and the process. Therefore henceforth we also use the notation

$$
W(\sigma(t), P)=\widehat{W}\left(\mathbf{E}(t), \breve{\mathbf{E}}^{t} ; \dot{\mathbf{E}}_{P}\right)=\widetilde{W}\left(\mathbf{E}(t), \mathbf{E}^{t} ; \dot{\mathbf{E}}_{P}\right)
$$


and view a process as a function $P: \Sigma_{w} \rightarrow \Sigma_{w}$ that associates with an initial state $\sigma^{i} \in \Sigma_{w}$ a final state $P \sigma^{i}=\sigma^{f} \in \Sigma_{w}$. The set of finite work processes $P$ will be denoted by $\Pi$, namely $P \in \Pi$ if the related $\dot{\mathbf{E}}_{P}$ satisfies Definition 3.3 .

4. Equivalent formulations of the maximum recoverable work. The maximum recoverable work is defined as follows

Definition 4.1. Given a state $\sigma \in \Sigma_{w}$, the maximum recoverable work $W_{R}$ starting from $\sigma$ is defined as

$$
W_{R}(\sigma):=\sup \{-W(\sigma, P): P \in \Pi\} .
$$

The name of "maximum recoverable work" for $W_{R}(\sigma)$ is to indicate that it is the maximum quantity of work we can "extract" from the material at the given state $\sigma$; in other words, it is the amount of energy that is available at $\sigma$.

Note that $W_{R}(\sigma)$ is a function of state and it is nonnegative, since the null process belongs to $\Pi$ and yields a null work.

Even if (4.1) is widely recognized as the definition of the maximum recoverable work, nevertheless in the literature alternative formulations have been used in order to obtain its expression in terms of the past histories, and consequently the explicit formula of the minimum free energy. Usually such alternative formulations are motivated by reasonable arguments, but not rigorously proved. For instance, Breuer and Onat in [1, 2] implicitly assume that $W_{R}$ is obtained by means of processes such that the final strain vanishes, namely,

$$
W_{R}(\sigma)=\sup \left\{-W(\sigma, P): P \in \Pi_{0}(\sigma)\right\}
$$

where

$$
\Pi_{0}(\sigma):=\left\{P \in \Pi: P \sigma=(\mathbf{0}, \mathbf{h}) \text { for some suitable } \mathbf{h} \in \Gamma_{w} / \Gamma_{w 0}\right\} .
$$

On the other hand, Day $([4,5])$ obtains $W_{R}$ by using processes such that the final strain has the same value as the initial one, viz.

$$
W_{R}(\sigma)=\frac{1}{2} \mathbb{G}_{\infty} \mathbf{E} \cdot \mathbf{E}+\sup \left\{-W(\sigma(t), P), P \in \Pi^{0}\right\},
$$

where

$$
\Pi^{0}:=\left\{P \in \Pi: \int_{0}^{d} \dot{\mathbf{E}}_{P}(t) d t=0\right\},
$$

and $d$ is the duration of the process. ${ }^{1}$ Finally, Golden in [17] finds the minimum free energy by maximizing the recoverable work in the form

$$
W_{R}(\sigma)=\sup \{S(\infty)-W(\sigma(t), P), P \in \Pi\},
$$

where $S(\infty)=\lim _{t \rightarrow \infty} S(t)$, and

$$
S(t)=\mathbf{T}(t) \cdot \mathbf{E}(t)-\frac{1}{2} \mathbb{G}_{0} \mathbf{E}(t) \cdot \mathbf{E}(t)
$$

turns out to be the opposite of the work done by a jump from $\mathbf{E}(t)$ to $\mathbf{0}$ (see also [9]).

\footnotetext{
${ }^{1}$ Actually, Day's statement is slightly different. Nevertheless, for our purpose it can be resumed to
} Eqs. (4.4-4.5). This will be made precise before Corollary 4.6. 
Since such formulations are quite different and since they must be related to the minimum free energy, which is supposed to be unique, one can ask whether they are equivalent.

A first proof, showing the equivalence between (4.1), (4.2) and (4.6), has been given in [9]. Here we extend such results to prove the equivalence between (4.4) and (4.1). Moreover, this result leads to a generalization of (4.4) that has been used in [13].

The proofs will be given without involving any topology or norm, so that the results are not affected by the choice of the topology for the description of the state space.

ThEOREM 4.2. For every state $\sigma \in \Sigma_{w}$, the maximum recoverable work $W_{R}(\sigma)$ defined in (4.1) is given by

$$
W_{R}(\sigma)=\sup \left\{-W(\sigma, P), P \in \Pi_{0}(\sigma)\right\},
$$

where $\Pi_{0}(\sigma)$ is given by $(4.3)$.

Before giving the proof we need a preliminary lemma.

LEMmA 4.3. Given a state $\sigma(t)$ related to the couple $\left(\mathbf{E}(t), \mathbf{E}^{t}\right)$, with $\mathbf{E}(t)=\mathbf{E}$, for every $\varepsilon>0$ there exist two suitable parameters $a, r$, such that the process $P_{a, r} \in \Pi_{0}(\sigma)$, of duration $a+r$ related to

$$
\mathbf{E}_{P}(\tau)= \begin{cases}\mathbf{E} & \text { for } \tau \in(0, a), \\ \mathbf{E}-\frac{\mathbf{E}}{r}(\tau-a) & \text { for } \tau \in(a, a+r],\end{cases}
$$

yields a work $W\left(\sigma(t), P_{a, r}\right)$ satisfying

$$
\left|W\left(\sigma(t), P_{a, r}\right)+\frac{1}{2} \mathbb{G}_{\infty} \mathbf{E} \cdot \mathbf{E}\right|<\varepsilon .
$$

Proof.

$$
\begin{aligned}
-W\left(\sigma(t), P_{a, r}\right)= & \int_{0}^{r} \mathbf{T}(t+a+\tau) d \tau \cdot \frac{\mathbf{E}}{r} \\
= & \frac{1}{2} \mathbb{G}_{0} \mathbf{E} \cdot \mathbf{E}+\int_{0}^{r} \int_{0}^{\tau} \dot{\mathbb{G}}(s)\left[\mathbf{E}-\frac{\mathbf{E}}{r}(\tau-s)\right] d s d \tau \cdot \frac{\mathbf{E}}{r} \\
& +\int_{0}^{r} \int_{\tau}^{\tau+a} \dot{\mathbb{G}}(s) d s d \tau \mathbf{E} \cdot \frac{\mathbf{E}}{r} \\
& +\int_{0}^{r} \int_{\tau+a}^{\infty} \dot{\mathbb{G}}(s) \mathbf{E}^{t}(s-a-\tau) d s d \tau \cdot \frac{\mathbf{E}}{r} .
\end{aligned}
$$

Integrating and making use of $(2.3)$, i.e., $\check{G}(t)=\mathbb{G}(t)-\mathbb{G}_{\infty}$, we have

$$
\begin{aligned}
& -W\left(\sigma(t), P_{a, r}\right)-\frac{1}{2} \mathbb{G}_{0} \mathbf{E} \cdot \mathbf{E}+\frac{1}{2} \check{\mathbb{G}}_{0} \mathbf{E} \cdot \mathbf{E}=\int_{0}^{r} \int_{0}^{\tau} \check{\mathbb{G}}(s) d s d \tau \frac{\mathbf{E}}{r} \cdot \frac{\mathbf{E}}{r} \\
& +\int_{0}^{r}[\check{\mathbb{G}}(\tau+a)-\check{\mathbb{G}}(\tau)] d \tau \mathbf{E} \cdot \frac{\mathbf{E}}{r}+\int_{0}^{r} \int_{0}^{\infty} \check{\mathbb{G}}(s+\tau+a) \mathbf{E}^{t}(s) d s d \tau \cdot \frac{\mathbf{E}}{r} .
\end{aligned}
$$

Observe that

$$
-W\left(\sigma(t), P_{a, r}\right)-\frac{1}{2} \mathbb{G}_{0} \mathbf{E} \cdot \mathbf{E}+\frac{1}{2} \check{\mathbb{G}}_{0} \mathbf{E} \cdot \mathbf{E}=-W\left(\sigma(t), P_{a, r}\right)-\frac{1}{2} \mathbb{G}_{\infty} \mathbf{E} \cdot \mathbf{E}
$$


Moreover, by virtue of $(2.4)$,

$$
\left|\int_{0}^{r} \int_{0}^{\tau} \check{\mathbb{G}}(s) d s d \tau \frac{\mathbf{E}}{r} \cdot \frac{\mathbf{E}}{r}+\int_{0}^{r}[\check{\mathbb{G}}(\tau+a)-\check{\mathbb{G}}(\tau)] d \tau \mathbf{E} \cdot \frac{\mathbf{E}}{r}\right|<\frac{M}{r}
$$

for some $M<\infty$. Moreover, by virtue of (3.1), we have

$$
\left|\int_{0}^{\infty} \dot{\mathbb{G}}(s+\tau+a) \mathbf{E}^{t}(s) d s\right|<\delta, \quad \forall(\tau+a)>a\left(\delta, \mathbf{E}^{t}\right)
$$

so that

$$
\left|\int_{0}^{r} \int_{0}^{\infty} \dot{\mathbb{G}}(s+\tau+a) \mathbf{E}^{t}(s) d s d \tau \cdot \frac{\mathbf{E}}{r}\right|<\delta|\mathbf{E}|, \quad \forall a>a\left(\delta, \mathbf{E}^{t}\right) .
$$

Choosing $r=\frac{2 M}{\varepsilon}, \delta=\frac{\varepsilon}{2|\mathbf{E}|}$ and $a>a\left(\delta, \mathbf{E}^{t}\right),(4.11)-(4.14)$ yield (4.10).

Proof of Theorem 4.2. Obviously, since $\Pi_{0}(\sigma) \subset \Pi$, it follows that

$$
W_{R}(\sigma)=\sup \{-W(\sigma, P): P \in \Pi\} \geq \sup \left\{-W(\sigma, P): P \in \Pi_{0}(\sigma)\right\} .
$$

In view of our hypothesis $W_{R}(\sigma)<\infty$, for every $\varepsilon>0$ there exists a process $P_{\varepsilon} \in \Pi$, of duration $d<\infty$, such that

$$
W_{R}(\sigma)-\frac{\varepsilon}{2}<-W\left(\sigma, P_{\varepsilon}\right) .
$$

Suppose that $P_{\varepsilon} \sigma=\left(\mathbf{E}_{\varepsilon}, \mathbf{h}\right)$ for some suitable $\mathbf{h} \in \Gamma_{w} / \Gamma_{w 0}$ and continue $P_{\varepsilon}$ with a process $P_{a, r}$ described by (4.9). Then, by virtue of (4.16) and Lemma 4.3, there exist two values of $a$ and $r$ such that

$$
-W\left(\sigma, P_{a, r} * P_{\varepsilon}\right)=-W\left(\sigma, P_{\varepsilon}\right)-W\left(P_{\varepsilon} \sigma, P_{a, r}\right)>W_{R}(\sigma)+\frac{1}{2} \mathbb{G}_{\infty} \mathbf{E}_{\varepsilon} \cdot \mathbf{E}_{\varepsilon}-\varepsilon .
$$

Finally, observe that $\left(P_{a, r} * P_{\varepsilon}\right) \in \Pi_{0}(\sigma)$ so that (4.17) and (2.8) imply

$$
W_{R}(\sigma) \leq \sup \left\{-W(\sigma, P): P \in \Pi_{0}(\sigma)\right\} .
$$

Inequalities (4.15) and (4.18) ensure (4.8).

An interesting alternative formulation of the maximum recoverable work is the following.

Theorem 4.4. For every state $\sigma \in \Sigma_{w}$, and for any fixed $\mathbf{E} \in S y m$, the maximum recoverable work $W_{R}(\sigma)$ defined in (4.1) is given by

$$
W_{R}(\sigma)=\frac{1}{2} \mathbb{G}_{\infty} \mathbf{E} \cdot \mathbf{E}+\sup \left\{-W(\sigma, P), P \in \Pi_{\mathbf{E}}(\sigma)\right\},
$$

where

$$
\Pi_{\mathbf{E}}(\sigma):=\left\{P \in \Pi: P \sigma=(\mathbf{E}, \mathbf{h}) \quad \text { for some suitable } \mathbf{h} \in \Gamma_{w} / \Gamma_{w 0}\right\} .
$$

The proof will be given with the aid of the following lemma.

Lemma 4.5. Given a state related to the couple $\left(\mathbf{0}, \mathbf{E}^{t}\right)$, for every $\varepsilon>0$ and every fixed strain $\mathbf{E} \in S y m$ there exist two suitable parameters $b, r$, such that the process $P_{b, r} \in \Pi_{\mathbf{E}}(\sigma)$, of duration $b+r$ related to

$$
\mathbf{E}_{P}(\tau)= \begin{cases}\mathbf{0} & \text { for } \tau \in(0, b) \\ \frac{\mathbf{E}}{r}(\tau-b) & \text { for } \tau \in(b, b+r]\end{cases}
$$


yields a work $W\left(\sigma(t), P_{b, r}\right)$ satisfying

$$
\left|W\left(\sigma(t), P_{b, r}\right)-\frac{1}{2} \mathbb{G}_{\infty} \mathbf{E} \cdot \mathbf{E}\right|<\varepsilon .
$$

Proof.

$$
\begin{aligned}
W\left(\sigma(t), P_{b, r}\right)= & \int_{0}^{r} \mathbf{T}(t+b+\tau) d \tau \cdot \frac{\mathbf{E}}{r} \\
= & \frac{1}{2} \mathbb{G}_{0} \mathbf{E} \cdot \mathbf{E}+\int_{0}^{r} \int_{0}^{\infty} \dot{\mathbb{G}}(s) \mathbf{E}(t+b+\tau-s) d s d \tau \cdot \frac{\mathbf{E}}{r} \\
= & \frac{1}{2} \mathbb{G}_{0} \mathbf{E} \cdot \mathbf{E}+\int_{0}^{r} \int_{0}^{\tau} \dot{\mathbb{G}}(s) \frac{\mathbf{E}}{r}(\tau-s) d s d \tau \cdot \frac{\mathbf{E}}{r} \\
& +\int_{0}^{r} \int_{0}^{\infty} \dot{\mathbb{G}}(s+b+\tau) \mathbf{E}^{t}(s) d s d \tau \cdot \frac{\mathbf{E}}{r} .
\end{aligned}
$$

Integrating (4.23) and making use of (2.3), we have

$$
\begin{aligned}
& W\left(\sigma(t), P_{b, r}\right)-\frac{1}{2} \mathbb{G}_{\infty} \mathbf{E} \cdot \mathbf{E} \\
& =\int_{0}^{r} \int_{0}^{\tau} \check{\mathbb{G}}(s) d s d \tau \frac{\mathbf{E}}{r} \cdot \frac{\mathbf{E}}{r}+\int_{0}^{r} \int_{0}^{\infty} \dot{\mathbb{G}}(s+\tau+a) \mathbf{E}^{t}(s) d s d \tau \cdot \frac{\mathbf{E}}{r} .
\end{aligned}
$$

Observe that, by virtue of $(2.4)$,

$$
\left|\int_{0}^{r} \int_{0}^{\tau} \check{G}(s) d s d \tau \frac{\mathbf{E}}{r} \cdot \frac{\mathbf{E}}{r}\right|<\frac{M}{r}
$$

for some $M<\infty$. Moreover, by virtue of (3.1), we have

$$
\left|\int_{0}^{\infty} \dot{\mathbb{G}}(s+\tau+b) \mathbf{E}^{t}(s) d s\right|<\delta, \quad \forall(\tau+b)>a\left(\delta, \mathbf{E}^{t}\right)
$$

so that

$$
\left|\int_{0}^{r} \int_{0}^{\infty} \dot{\mathbb{G}}(s+\tau+b) \mathbf{E}^{t}(s) d s d \tau \cdot \frac{\mathbf{E}}{r}\right|<\delta|\mathbf{E}|, \quad \forall b>a\left(\delta, \mathbf{E}^{t}\right) .
$$

Choosing $r=\frac{2 M}{\varepsilon}, \delta=\frac{\varepsilon}{2|\mathbf{E}|}$ and $a>a\left(\delta, \mathbf{E}^{t}\right),(4.24)-(4.26)$ yield (4.19).

Proof of Theorem 4.4. Denoting by $\mathcal{W}_{0}(\sigma)$ and $\mathcal{W}_{\mathbf{E}}(\sigma)$ the following sets:

$$
\begin{aligned}
\mathcal{W}_{0}(\sigma) & :=\left\{-W(\sigma, P) ; P \in \Pi_{0}(\sigma)\right\}, \\
\mathcal{W}_{\mathbf{E}}(\sigma) & :=\left\{\frac{1}{2} \mathbb{G}_{\infty} \mathbf{E} \cdot \mathbf{E}-W(\sigma, P) ; P \in \Pi_{\mathbf{E}}(\sigma)\right\},
\end{aligned}
$$

observe that, for every $P_{\mathbf{E}} \in \Pi_{\mathbf{E}}(\sigma)$, there exists a sequence of $P_{n} \in \Pi_{0}(\sigma)$ such that

$$
-\lim _{n \rightarrow \infty} W\left(\sigma, P_{n}\right)=\frac{1}{2} \mathbb{G}_{\infty} \mathbf{E} \cdot \mathbf{E}-W\left(\sigma, P_{\mathbf{E}}\right) .
$$

In fact, if we continue any process $P_{\mathbf{E}} \in \Pi_{\mathbf{E}}(\sigma)$, yielding a work $W\left(\sigma, P_{\mathbf{E}}\right)$, with the process $P_{a, r}$ given by (4.9), we obtain a process $P_{a, r} * P_{\mathbf{E}} \in \Pi_{0}(\sigma)$. Moreover, (4.10) and the following

$$
W\left(\sigma, P_{a, r} * P_{\mathbf{E}}\right)=W\left(\sigma, P_{\mathbf{E}}\right)+W\left(P_{\mathbf{E}} \sigma, P_{a, r}\right)
$$


imply that for any $\varepsilon>0$, there exists a process $P_{a, r} * P_{\mathbf{E}} \in \Pi_{0}(\sigma)$ such that

$$
\left|W\left(\sigma, P_{a, r} * P_{\mathbf{E}}\right)-W\left(\sigma, P_{\mathbf{E}}\right)+\frac{1}{2} \mathbb{G}_{\infty} \mathbf{E} \cdot \mathbf{E}\right|<\varepsilon,
$$

from which the limit (4.29) follows. This implies that

$$
\mathcal{W}_{\mathbf{E}}(\sigma) \subseteq \overline{\mathcal{W}_{0}(\sigma)}
$$

On the other hand, for every $P_{0} \in \Pi_{0}(\sigma)$, there exists a sequence of $P_{n} \in \Pi_{\mathbf{E}}(\sigma)$ such that

$$
\frac{1}{2} \mathbb{G}_{\infty} \mathbf{E} \cdot \mathbf{E}-\lim _{n \rightarrow \infty} W\left(\sigma, P_{n}\right)=-W\left(\sigma, P_{0}\right) .
$$

In fact, if we continue any process $P_{0} \in \Pi_{0}(\sigma)$, yielding a work $W\left(\sigma, P_{0}\right)$, with the process $P_{b, r}$ given by (4.21), we obtain $P_{b, r} * P_{0} \in \Pi_{\mathbf{E}}(\sigma)$. Moreover, relation (4.22) and the following

$$
W\left(\sigma, P_{b, r} * P_{0}\right)=W\left(\sigma, P_{0}\right)+W\left(P_{0} \sigma, P_{b, r}\right)
$$

imply that for any $\varepsilon>0$, there exists a process $P_{b, r} * P_{0} \in \Pi_{\mathbf{E}}(\sigma)$ such that

$$
\left|W\left(\sigma, P_{b, r} * P_{0}\right)-W\left(\sigma, P_{0}\right)-\frac{1}{2} \mathbb{G}_{\infty} \mathbf{E} \cdot \mathbf{E}\right|<\varepsilon \text {. }
$$

This implies that

$$
\mathcal{W}_{0}(\sigma) \subseteq \overline{\mathcal{W}_{\mathbf{E}}(\sigma)}
$$

Equations (4.30) and (4.31) imply $\overline{\mathcal{W}_{0}(\sigma)}=\overline{\mathcal{W}_{\mathbf{E}}(\sigma)}$ so that $\sup \mathcal{W}_{0}(\sigma)=\sup \mathcal{W}_{\mathbf{E}}(\sigma)$. By virtue of Theorem 4.2 the thesis follows.

Theorem 4.4 is a generalization of the formulation of the maximum recoverable work given by Day. For sake of precision, the maximum recoverable work $W_{R}^{(D)}$ defined by Day is just the quantity $W_{R}^{(D)}(\sigma(t))=\sup \left\{-W(\sigma(t), P), P \in \Pi^{0}\right\}$. However, since any free energy $\psi$ evaluated at the constant strain history $\left(\mathbf{E}, \mathbf{E}^{\dagger}\right)$ gives $\psi=\frac{1}{2} \mathbb{G}_{\infty} \mathbf{E} \cdot \mathbf{E}$, he stated that the minimum free energy $\psi_{m}$ relative to the strain history $\left(\mathbf{E}(t), \mathbf{E}^{t}\right)$ is given by $\psi_{m}(t)=\frac{1}{2} \mathbb{G}_{\infty} \mathbf{E}(t) \cdot \mathbf{E}(t)+W_{R}^{(D)}(\sigma(t))$. Therefore, for our purpose (see (5.1)), Day's point of view can be stated as follows.

Corollary 4.6. For every state $\sigma \in \Sigma_{w}$, and for any fixed $\mathbf{E} \in S y m$, the maximum recoverable work $W_{R}(\sigma)$ defined in (4.1) is also given by

$$
W_{R}(\sigma)=\frac{1}{2} \mathbb{G}_{\infty} \mathbf{E}(t) \cdot \mathbf{E}(t)+\sup \left\{-W(\sigma, P), P \in \Pi^{0}\right\},
$$

where $\Pi^{0}$ is defined by (4.5).

Moreover, Theorem 4.4 may be generalized in the following form that has been used by Fabrizio and Golden [13]. Let $\phi(t)=\frac{1}{2} \mathbb{G}_{\infty} \mathbf{E}(t) \cdot \mathbf{E}(t)$ and $\phi(\infty)=\lim _{t \rightarrow \infty} \phi(t)$. Observe that in our context the limit $\phi(\infty)$ does exist, since any process $P \in \Pi$ has finite duration. Therefore, by continuing any process $P$ of duration $d$ with the null process, the work done does not change and the ensuing strain is such that $\mathbf{E}(t)=\mathbf{E}(d), \forall t \geq d$. Thus the limit $\mathbf{E}_{\infty}=\lim _{t \rightarrow \infty} \mathbf{E}(t)$ exists and corresponds to the final strain yielded by $P$, whereas $\phi(\infty)=\frac{1}{2} \mathbb{G}_{\infty} \mathbf{E}_{\infty} \cdot \mathbf{E}_{\infty}$. 
Corollary 4.7. For every state $\sigma \in \Sigma_{w}$, the maximum recoverable work $W_{R}(\sigma)$ defined in (4.1) is given by

$$
W_{R}(\sigma)=\sup \{\phi(\infty)-W(\sigma, P), P \in \Pi\} .
$$

Proof. First observe that $P \in \Pi_{0}(\sigma)$, of duration $d<\infty$, continued with the null process yields a strain that vanishes at infinity, i.e., $\mathbf{E}_{\infty}=0$, so that $\phi(\infty)=0$. Therefore, letting $\mathcal{W}_{0}(\sigma)$ and $\mathcal{W}_{\infty}(\sigma)$ be defined by $(4.27)$ and by

$$
\mathcal{W}_{\infty}(\sigma):=\{\phi(\infty)-W(\sigma, P) ; P \in \Pi\}
$$

we have

$$
\mathcal{W}_{0}(\sigma) \subset \mathcal{W}_{\infty}(\sigma)
$$

Now fix a state $\sigma(t)$ and a process $P \in \Pi$ yielding a final strain such that

$$
\lim _{\tau \rightarrow \infty} \mathbf{E}(\tau)=\mathbf{E}_{\infty} \neq 0 .
$$

Let $P_{d}$ be the truncation of $P$ up to the time $d$, i.e., the process of duration $d$ continuing $\sigma(t)$ with the strain $\mathbf{E}_{d}$ such that $\mathbf{E}_{d}(\tau)=\mathbf{E}(\tau), \tau \in[t, t+d)$. Then since

$$
\lim _{d \rightarrow \infty} W\left(\sigma, P_{d}\right)=W(\sigma, P), \quad \lim _{d \rightarrow \infty} \frac{1}{2} \mathbb{G}_{\infty} \mathbf{E}_{d} \cdot \mathbf{E}_{d}=\frac{1}{2} \mathbb{G}_{\infty} \mathbf{E}_{\infty} \cdot \mathbf{E}_{\infty}
$$

for every $\varepsilon$, there exists a suitable $d_{\varepsilon}$ such that

$$
\left|W\left(\sigma, P_{d}\right)-W(\sigma, P)\right|<\frac{\varepsilon}{3}, \quad\left|\frac{1}{2} \mathbb{G}_{\infty} \mathbf{E}_{d} \cdot \mathbf{E}_{d}-\frac{1}{2} \mathbb{G}_{\infty} \mathbf{E}_{\infty} \cdot \mathbf{E}_{\infty}\right|<\frac{\varepsilon}{3}
$$

holds for every $d>d_{\varepsilon}$.

Now we consider a process $P_{d, a, r}=P_{a, r} * P_{d} \in \Pi_{0}(\sigma(t))$, with $d$ such that (4.36) is satisfied and $P_{a, r}$ is described in Lemma 4.3, related to the strain (4.9) with $\mathbf{E}=\mathbf{E}_{d}$ and $a, r$ such that $P_{a, r}$ satisfies (4.10) with $\varepsilon$ replaced by $\frac{1}{3} \varepsilon$.

Then, for every $\varepsilon>0$, there exists a process $P_{\varepsilon} \in \Pi_{0}(\sigma(t))$ such that

$$
\left|\phi(\infty)-W(\sigma, P)+W\left(\sigma, P_{\varepsilon}\right)\right|<\varepsilon .
$$

In fact, considering $P_{\varepsilon}=P_{d, a, r}$ we have

$$
W\left(\sigma, P_{\varepsilon}\right)=W\left(\sigma, P_{a, r} * P_{d}\right)=W\left(\sigma, P_{d}\right)+W\left(P_{d} \sigma, P_{a, r}\right)
$$

and

$$
\begin{aligned}
|\phi(\infty)-W(\sigma, P)+W(\sigma, P \varepsilon)|= & \left|\phi(\infty)-W(\sigma, P)+W\left(\sigma, P_{d}\right)+W\left(P_{d} \sigma, P_{a, r}\right)\right| \\
< & \left|\phi(\infty)-\frac{1}{2} \mathbb{G}_{\infty} \mathbf{E}_{d} \cdot \mathbf{E}_{d}\right|+\left|W(\sigma, P)-W\left(\sigma, P_{d}\right)\right| \\
& +\left|W\left(P_{d} \sigma, P_{a, r}\right)+\frac{1}{2} \mathbb{G}_{\infty} \mathbf{E}_{d} \cdot \mathbf{E}_{d}\right|<\varepsilon .
\end{aligned}
$$

Estimate (4.37) means that for each fixed $\sigma \in \Sigma_{w}$ and $P \in \Pi$ yielding a work $W(\sigma, P)$, there exists a sequence of processes $P_{d_{n}, a_{n}, r_{n}} \in \Pi_{0}(\sigma)$ yielding works $W\left(\sigma, P_{d_{n}, a_{n}, r_{n}}\right) \in$ $\mathcal{W}_{0}(\sigma)$ such that

$$
\lim _{n \rightarrow \infty} \widehat{W}\left(\sigma, P_{d_{n}, a_{n}, r_{n}}\right)=W(\sigma, P)-\phi(\infty)
$$


and hence

$$
\mathcal{W}_{\infty}(\sigma) \subseteq \overline{\mathcal{W}_{0}(\sigma)}
$$

Inclusions (4.35) and (4.38) imply that

$$
\sup \mathcal{W}_{\infty}(\sigma)=\sup \mathcal{W}_{0}(\sigma)
$$

By virtue of definitions (4.34) and Lemma 4.3, the thesis is proved.

We conclude this section by recalling the equivalent formulation for $W_{R}$ used by Golden in [17]. Such a formulation may be resumed as follows.

THEOREM 4.8. The maximum recoverable work $W_{R}(\sigma(t))$ is given by

$$
W_{R}(\sigma(t))=\sup \{S(\infty)-W(\sigma(t), P), P \in \Pi\},
$$

where $S(\infty)=\lim _{t \rightarrow \infty} S(t)$ and $S(t)$ is defined by (4.7).

Proof. Any process $P \in \Pi_{0}(\sigma)$ yields a final strain $\mathbf{E}_{\infty}=0$, so that $S(\infty)=0$. Therefore, letting $\mathcal{W}_{0}(\sigma)$ and $\mathcal{W}_{\infty}^{\prime}(\sigma)$ be defined by $(4.27)$ and by

$$
\mathcal{W}_{\infty}^{\prime}(\sigma):=\{S(\infty)-W(\sigma, P) ; P \in \Pi\}
$$

we have

$$
\mathcal{W}_{0}(\sigma) \subset \mathcal{W}_{\infty}^{\prime}(\sigma)
$$

Now consider the strain $\mathbf{E}(\tau), \tau \in \mathcal{R}$, related to $\sigma(t)$ continued with a process $P \in \Pi$ and such that $\lim _{\tau \rightarrow \infty} \mathbf{E}(\tau)=\mathbf{E}_{\infty} \neq 0$. Let $\mathbf{E}_{d}$. denote the truncation of $\mathbf{E}$ at the time $d>t$, continued with the "jump"-process yielding

$$
\lim _{\tau \rightarrow d^{-}} \mathbf{E}_{d}(\tau)=\mathbf{E}(d), \quad \mathbf{E}_{d}(\tau)=0, \quad \tau \geq d,
$$

and call $P_{d}$ the related process. The work done on $P_{d}$ is

$$
\begin{aligned}
W\left(\sigma(t), P_{d}\right) & =\int_{t}^{d} \mathbf{T}(\tau) \cdot \dot{\mathbf{E}}(\tau) d \tau-\frac{1}{2}\left[\mathbf{T}\left(d^{+}\right)+\mathbf{T}\left(d^{-}\right)\right] \cdot \mathbf{E}(d) \\
& =\int_{t}^{d} \mathbf{T}(\tau) \cdot \dot{\mathbf{E}}(\tau) d \tau-S(d) .
\end{aligned}
$$

Since

$$
\lim _{d \rightarrow \infty} W\left(\sigma(t), P_{d}\right)=W(\sigma(t), P)-S(\infty)
$$

for every $P \in \Pi$ we can find a family of processes $P_{d} \in \Pi_{0}(\sigma(t))$ such that

$$
\lim _{d \rightarrow \infty} W\left(\sigma(t), P_{d}\right)=W(\sigma(t), P)-S(\infty) .
$$

Therefore

$$
\mathcal{W}_{\infty}^{\prime}(\sigma(t)) \subset \overline{\mathcal{W}_{0}(\sigma(t))}
$$

(4.40) and (4.44) imply (4.39). 
5. An expression for the minimum free energy. The minimum free energy has been shown to be given by the maximum recoverable work, viz.

$$
\psi_{m}(\sigma)=W_{R}(\sigma)
$$

The problem of finding an expression for the maximum recoverable work, and hence for the minimum free energy, in the general case for a viscoelastic material has been considered first by Day in [4]. However, such works do not completely solve the problem because the exhibited final expression is not in terms of the given strain, but in terms of the "reversible extension", that is, the "optimal future continuation" of the strain yielding the maximum recoverable work. Now, such a reversible extension can be written in terms of the given strain by solving a Wiener-Hopf equation of the first kind. Unfortunately, this type of integral equation is not solvable unless particular properties of the integral kernel are specified. Therefore, Day's formula remains an interesting characterization, but not an explicit formula for the minimum free energy. Recently Golden [17] has provided an explicit expression for the minimum free energy in the scalar case by using a variational technique. Such a method has been extended to the tensorial case in [9]. However, the results on factorization for the memory kernel, which turn out to be crucial both in [17] and [9], may also be applied to Day formulation to yield an explicit formula for the maximum recoverable work in terms of the given strain, and hence for the minimum free energy. This is just the aim of this section, namely, to give an explicit characterization of $W_{R}(\sigma)$, and hence of $\psi_{m}(\sigma)$ starting from relation (4.19) of Corollary 4.6.

Consider a fixed time instant $t$ and a strain history $\left(\mathbf{E}(t), \breve{\mathbf{E}}^{t}\right)$ represented by a state $\sigma(t) \in \Sigma_{w}$. The work done on a process $P \in \Pi^{0}$, related to the strain $\mathbf{E}_{P}:(0, \infty) \rightarrow S y m$, is given by (3.16) with $\mathbf{E}_{0}(d)=\mathbf{0}$, viz.

$$
\widehat{W}\left(\mathbf{E}(t), \breve{\mathbf{E}}^{t} ; \dot{\mathbf{E}}_{P}\right)=\int_{0}^{\infty}\left[\frac{1}{2} \int_{0}^{\infty} \check{\widetilde{G}}(|\tau-s|) \dot{\mathbf{E}}_{P}(s) d s-\breve{\mathbf{I}}\left(\tau, \breve{\mathbf{E}}^{t}\right)\right] \cdot \dot{\mathbf{E}}_{P}(\tau) d \tau .
$$

The "optimal process" $P^{(m)}$ will be the one related to the future strain $\mathbf{E}^{(m)}:(0, \infty) \rightarrow$ Sym providing the maximum recoverable work, namely, such that

$$
W_{R}(\sigma(t))=\frac{1}{2} \mathbb{G}_{\infty} \mathbf{E}(t) \cdot \mathbf{E}(t)-\widehat{W}\left(\mathbf{E}(t), \breve{\mathbf{E}}^{t} ; \dot{\mathbf{E}}^{(m)}\right) .
$$

For each $\dot{\mathbf{E}}_{P}$ we put $\dot{\mathbf{E}}_{P}(s)=\dot{\mathbf{E}}^{(m)}(s)+\mathbf{h}(s)$ for a suitable $\mathbf{h}: \mathcal{R}^{-} \rightarrow$ Sym. Therefore $\widehat{W}\left(\mathbf{E}(t), \breve{\mathbf{E}}^{t} ; \dot{\mathbf{E}}_{P}\right)$ is minimized in correspondence of $\mathbf{E}^{(m)}$ satisfying

$$
\int_{0}^{\infty} \check{\widetilde{G}}(|\tau-s|) \dot{\mathbf{E}}^{(m)}(s) d s=\breve{\mathbf{I}}\left(\tau, \breve{\mathbf{E}}^{t}\right), \quad \tau \in \mathcal{R}^{+} .
$$

Such an equation turns out to be a Wiener-Hopf equation of the first kind, which is not solvable in the general case. Nevertheless, the thermodynamic properties of the integral kernel and some theorems on factorization will allow us to determine the solution $\mathbf{E}^{(m)}$ of (5.4).

Let us introduce a new function $\mathbf{r}: \mathcal{R}^{-} \rightarrow$ Sym, defined as

$$
\mathbf{r}(\tau)=\int_{-\infty}^{\infty} \check{\mathbb{G}}(|\tau-s|) \dot{\mathbf{E}}^{(m)}(s) d s, \quad \tau \in \mathcal{R}^{-},
$$


which, added to (5.4), yields

$$
\int_{-\infty}^{\infty} \check{\mathbb{G}}(|\tau-s|) \dot{\mathbf{E}}^{(m)}(s) d s=\breve{\mathbf{I}}\left(\tau, \breve{\mathbf{E}}^{t}\right)+\mathbf{r}(\tau), \quad \tau \in \mathcal{R},
$$

where $\operatorname{supp}\left(\dot{\mathbf{E}}^{(m)}\right) \subseteq \mathcal{R}^{+}, \operatorname{supp}\left(\breve{\mathbf{I}}\left(\cdot, \breve{\mathbf{E}}^{t}\right)\right) \subseteq \mathcal{R}^{+}$and $\operatorname{supp}(\mathbf{r}) \subseteq \mathcal{R}^{-}$. The Fourier transform of (5.5) gives

$$
2 \check{\mathbb{G}}_{c}(\omega) \dot{\mathbf{E}}_{+}^{(m)}(\omega)=\breve{\mathbf{I}}_{+}^{t}(\omega)+\mathbf{r}_{-}(\omega),
$$

where $\breve{\mathbf{I}}_{+}^{t}(\omega)=\breve{\mathbf{I}}_{+}\left(\omega, \breve{\mathbf{E}}^{t}\right)=\int_{0}^{\infty} \breve{\mathbf{I}}\left(\tau, \breve{\mathbf{E}}^{t}\right) e^{-i \omega \tau} d \tau$.

It is worth recalling that we can apply results of [16] to fourth-order tensor-valued functions (see Appendix). In particular, the tensor $\mathbb{K}(\omega)=\left(1+\omega^{2}\right) \check{\mathbb{G}}_{c}(\omega)$ can be factorized as

$$
\mathbb{K}(\omega)=\mathbb{K}_{(+)}(\omega) \mathbb{K}_{(-)}(\omega),
$$

where henceforth the subscript $\left(_{ \pm}\right.$indicates that the function $f_{( \pm)}(z)$ has zeroes and singularities only for $z \in \Omega^{ \pm}$. In particular, $\mathbb{K}(\omega)$ has no zero for every real $\omega,-\infty \leq$ $\omega \leq \infty$. Moreover, $\check{\mathbb{G}}_{c}(\omega)$ can be factorized too as follows:

$$
\check{\mathbb{G}}_{c}(\omega)=\check{\mathbb{G}}_{(+)}(\omega) \check{\mathbb{G}}_{(-)}(\omega), \quad \check{\mathbb{G}}_{( \pm)}(\omega)=\frac{1}{1 \pm i \omega} \mathbb{K}_{ \pm}(\omega) .
$$

Factorization (5.7) allows us to rewrite (5.6) as follows:

$$
\check{\mathbb{G}}_{(+)}(\omega) \dot{\mathbf{E}}_{+}^{(m)}(\omega)=\frac{1}{2} \check{\mathbb{G}}_{(-)}^{-1}(\omega) \breve{\mathbf{I}}_{+}^{t}(\omega)+\frac{1}{2} \check{\mathbb{G}}_{(-)}^{-1}(\omega) \mathbf{r}_{-}(\omega)
$$

Applying the Plemelj formulae, the quantity $\breve{\mathbb{G}}_{(-)}^{-1}(\omega) \breve{\mathbf{I}}_{+}^{t}(\omega)$ may be rewritten as

$$
\frac{1}{2} \check{\mathbb{G}}_{(-)}^{-1}(\omega) \breve{\mathbf{I}}_{+}^{t}(\omega)=\mathbf{p}_{(-)}^{t}(\omega)-\mathbf{p}_{(+)}^{t}(\omega)
$$

where $\mathbf{p}_{( \pm)}^{t}(z)$ has zeroes and singularities for $z \in \Omega^{ \pm}$and they are defined by

$$
\mathbf{p}^{t}(z):=\frac{1}{4 \pi i} \int_{-\infty}^{\infty} \frac{\check{\mathbb{G}}_{(-)}^{-1}(\omega) \breve{\mathbf{I}}_{+}^{t}(\omega)}{z-\omega} d \omega, \quad \mathbf{p}_{( \pm)}^{t}(\omega):=\lim _{\alpha \rightarrow 0 \mp} \mathbf{p}^{t}(\omega+i \alpha) .
$$

Therefore, we obtain

$$
\check{\mathbb{G}}_{(+)}(\omega) \dot{\mathbf{E}}_{+}^{(m)}(\omega)=-\mathbf{p}_{(+)}^{t}(\omega)+\mathbf{p}_{(-)}^{t}(\omega)+\frac{1}{2} \check{\mathbb{G}}_{(-)}^{-1}(\omega) \mathbf{r}_{-}(\omega) .
$$

Observe that the quantities $\check{\mathbb{G}}_{(+)}(z) \dot{\mathbf{E}}_{+}^{(m)}(z)$ and $\mathbf{p}_{(+)}^{t}(z)$ are analytic for $z \in \Omega^{-}$, whereas $\mathbf{p}_{(-)}^{t}(z)$ and $\check{\mathbb{G}}_{(-)}^{-1}(\omega) \mathbf{r}_{-}(\omega)$ are analytic for $z \in \Omega^{+}$. Therefore, the quantity

$$
\mathbf{J}(\omega)=\check{\mathbb{G}}_{(+)}(\omega) \dot{\mathbf{E}}_{+}^{(m)}(\omega)+\mathbf{p}_{(+)}^{t}(\omega)=\mathbf{p}_{(-)}^{t}(\omega)+\frac{1}{2} \check{\mathbb{G}}_{(-)}^{-1}(\omega) \mathbf{r}_{-}(\omega)
$$

has analytic extension on the whole complex plane. Since $\mathbf{J}$ vanishes at infinity it must be zero so that

$$
\begin{aligned}
\dot{\mathbf{E}}_{+}^{(m)}(\omega) & =-\check{\mathbb{G}}_{(+)}(\omega)^{-1} \mathbf{p}_{(+)}^{t}(\omega) \\
\mathbf{p}_{(-)}^{t}(\omega) & =-\frac{1}{2} \check{\mathbb{G}}_{(-)}^{-1}(\omega) \mathbf{r}_{-}(\omega)
\end{aligned}
$$


It is worth noting that the process $P^{(m)}$ related to $\mathbf{E}^{(m)}$ does not belong to $\Pi^{0}$ but may be constructed as the limit of a sequence of processes $P_{n}$ of $\Pi^{0}$.

REMARK 5.1. Since $\mathbf{p}_{(+)}^{t}(\omega)=O(1 / \omega)$ as $|\omega| \rightarrow \infty$ and $\lim _{\omega \rightarrow \infty} \check{\mathbb{G}}_{(+)}(\omega)=$ $\frac{1}{i \omega}[-\dot{\mathbb{G}}(0)]^{1 / 2}$, it follows that

$$
\lim _{\omega \rightarrow \infty} \dot{\mathbf{E}}_{+}^{(m)}(\omega) \neq \mathbf{0}
$$

Therefore $\dot{\mathbf{E}}^{(m)}(\tau)$ has an initial singularity as $\tau \rightarrow 0^{+}$. This agrees with the property of the optimal continuation that must have an initial discontinuity as $\tau \rightarrow 0^{+}$.

REMARK 5.2. Observe that $\check{\mathbb{G}}_{(+)}(0) \neq 0$, so that

$$
\mathbf{E}^{(m)}(\infty)-\mathbf{E}^{(m)}(0)=\int_{0}^{\infty} \dot{\mathbf{E}}^{(m)}(\tau) d \tau=\dot{\mathbf{E}}_{+}^{(m)}(0)=-\check{\mathbb{G}}_{(+)}(0)^{-1} \mathbf{p}_{(+)}^{t}(0)
$$

Therefore, the solution $\dot{\mathbf{E}}^{(m)}$ of (5.4) tends to the finite limit

$$
\lim _{\tau \rightarrow \infty} \mathbf{E}^{(m)}(\tau)=\mathbf{E}^{(m)}(\infty)=\mathbf{E}(t)-\check{\mathbb{G}}_{(+)}(0)^{-1} \mathbf{p}_{(+)}^{t}(0)
$$

The substitution of (5.11) into (3), taking account of (4.36), yields

$$
\begin{aligned}
W_{R}(\sigma) & =\frac{1}{2} \mathbb{G}_{\infty} \mathbf{E}(t) \cdot \mathbf{E}(t)+\frac{1}{2} \int_{0}^{\infty} \int_{0}^{\infty} \check{\mathbb{G}}(|\tau-s|) \dot{\mathbf{E}}^{(m)}(s) \cdot \dot{\mathbf{E}}^{(m)}(\tau) d s d \tau \\
& =\frac{1}{2} \mathbb{G}_{\infty} \mathbf{E}(t) \cdot \mathbf{E}(t)+\frac{1}{2 \pi} \int_{-\infty}^{\infty} \check{\mathbb{G}}_{c}(\omega) \dot{\mathbf{E}}_{+}^{(m)}(\omega) \cdot \overline{\dot{\mathbf{E}}_{+}^{(m)}(\omega)} d \omega \\
& =\frac{1}{2} \mathbb{G}_{\infty} \mathbf{E}(t) \cdot \mathbf{E}(t)+\frac{1}{2 \pi} \int_{-\infty}^{\infty}\left|\mathbf{p}_{(+)}^{t}(\omega)\right|^{2} d \omega
\end{aligned}
$$

Therefore, in view of (5.1), the minimum free energy takes the form

$$
\psi_{m}(\sigma(t))=\hat{\psi}_{m}\left(\mathbf{E}(t), \mathbf{p}_{(+)}^{t}\right)=\frac{1}{2} \mathbb{G}_{\infty} \mathbf{E}(t) \cdot \mathbf{E}(t)+\frac{1}{2 \pi} \int_{-\infty}^{\infty}\left|\mathbf{p}_{(+)}^{t}(\omega)\right|^{2} d \omega .
$$

Actually, expression (5.13) suggests that the couple $\left(\mathbf{E}(t), \mathbf{p}_{(+)}^{t}\right)$ provides an explicit representation of the state $\sigma(t) \in \Sigma_{w}$; this question will be considered in the next section.

Now we conclude the section by comparing expression (5.13) for the minimum free energy with the ones already found in [17] and [9]. Let us exploit the relation between $\mathbf{p}_{(+)}^{t}(\omega)$ and $\breve{\mathbf{E}}^{t}$. We identify $\breve{\mathbf{E}}^{t}$ with its causal extension (viz. $\breve{\mathbf{E}}^{t}(\tau)=0$ for $\tau<0$ ) and consider the odd extension $\dot{\mathbb{G}}^{(d)}$ of $\dot{\mathbb{G}}$, viz.

$$
\dot{\mathbb{G}}^{(d)}(\xi)=\left\{\begin{array}{ll}
\dot{\mathbb{G}}(\xi) & \text { for } \xi \geq 0 \\
-\dot{\mathbb{G}}(-\xi) & \text { for } \xi<0
\end{array} \quad \text { so that } \quad \dot{\mathbb{G}}_{F}^{(d)}(\omega)=-2 i \omega \dot{\mathbb{G}}_{s}(\omega) .\right.
$$

Then (3.14) can be rewritten as

$$
\breve{\mathbf{I}}\left(\tau, \breve{\mathbf{E}}^{t}\right)=-\int_{-\infty}^{\infty} \dot{\mathbb{G}}^{(d)}(\tau+s) \breve{\mathbf{E}}^{t}(s) d s, \quad \tau \geq 0 .
$$

Moreover, let $\breve{\mathbf{I}}^{(n)}\left(\cdot, \breve{\mathbf{E}}^{t}\right): \mathcal{R}^{-} \rightarrow$ Sym be defined as

$$
\breve{\mathbf{I}}^{(n)}\left(\tau, \breve{\mathbf{E}}^{t}\right)=-\int_{-\infty}^{\infty} \dot{\mathbb{G}}^{(d)}(\tau+s) \breve{\mathbf{E}}^{t}(s) d s, \quad \tau<0,
$$


and consider the extension $\breve{\mathbf{I}}^{(R)}\left(\cdot, \breve{\mathbf{E}}^{t}\right): \mathcal{R} \rightarrow$ Sym of $\breve{\mathbf{I}}\left(\tau, \breve{\mathbf{E}}^{t}\right)$ to the real line as follows:

$$
\breve{\mathbf{I}}^{(R)}\left(\tau, \breve{\mathbf{E}}^{t}\right)=-\int_{-\infty}^{\infty} \dot{\mathbb{G}}^{(d)}(\tau+s) \breve{\mathbf{E}}^{t}(s) d s= \begin{cases}\breve{\mathbf{I}}\left(\tau, \breve{\mathbf{E}}^{t}\right) & \text { for } \tau \geq 0, \\ \breve{\mathbf{I}}^{(n)}\left(\tau, \breve{\mathbf{E}}^{t}\right) & \text { for } \tau<0 .\end{cases}
$$

Introducing the variable $\breve{\mathbf{E}}_{n}^{t}(s)=\breve{\mathbf{E}}^{t}(-s), s \leq 0$ and the ensuing extension $\breve{\mathbf{E}}_{n}^{t}(s)=0$ for $s>0$, we have

$$
\breve{\mathbf{I}}^{(R)}\left(\tau, \breve{\mathbf{E}}^{t}\right)=-\int_{-\infty}^{\infty} \dot{\mathbb{G}}^{(d)}(\tau-s) \breve{\mathbf{E}}_{n}^{t}(s) d s,
$$

so that

$$
\breve{\mathbf{I}}_{F}^{(R)}\left(\omega, \breve{\mathbf{E}}^{t}\right)=2 i \dot{\mathbb{G}}_{s}(\omega) \breve{\mathbf{E}}_{n-}^{t}(\omega)=2 i \dot{\mathbb{G}}_{s}(\omega) \overline{\breve{\mathbf{E}}_{+}^{t}(\omega)}
$$

By virtue of (5.16), we have the property

$$
\breve{\mathbf{I}}_{F}^{(R)}\left(\omega, \breve{\mathbf{E}}^{t}\right)=\breve{\mathbf{I}}_{+}\left(\omega, \breve{\mathbf{E}}^{t}\right)+\breve{\mathbf{I}}_{-}^{(n)}\left(\omega, \breve{\mathbf{E}}^{t}\right),
$$

so that

$$
\frac{1}{2} \check{\mathbb{G}}_{(-)}^{-1}(\omega) \breve{\mathbf{I}}_{F}^{(R)}\left(\omega, \breve{\mathbf{E}}^{t}\right)=\frac{1}{2} \check{\mathbb{G}}_{(-)}^{-1}(\omega) \breve{\mathbf{I}}_{+}\left(\omega, \breve{\mathbf{E}}^{t}\right)+\frac{1}{2} \check{\mathbb{G}}_{(-)}^{-1}(\omega) \breve{\mathbf{I}}_{-}^{(n)}\left(\omega, \breve{\mathbf{E}}^{t}\right)
$$

By virtue of (5.8) we have

$$
\frac{1}{2} \check{\mathbb{G}}_{(-)}^{-1}(\omega) \breve{\mathbf{I}}_{F}^{(R)}\left(\omega, \breve{\mathbf{E}}^{t}\right)=\mathbf{p}_{(-)}^{t}(\omega)-\mathbf{p}_{(+)}^{t}(\omega)+\frac{1}{2} \check{\mathbb{G}}_{(-)}^{-1}(\omega) \breve{\mathbf{I}}_{-}^{(n)}\left(\omega, \breve{\mathbf{E}}^{t}\right)
$$

On the other hand, the quantity $\frac{1}{2} \check{\mathbb{G}}_{(-)}^{-1}(\omega) \breve{\mathbf{I}}_{F}^{(R)}\left(\omega, \breve{\mathbf{E}}^{t}\right)$ may be written by using the Plemelj formulae as follows:

$$
\frac{1}{2} \check{\mathbb{G}}_{(-)}^{-1}(\omega) \breve{\mathbf{I}}_{F}^{(R)}\left(\omega, \breve{\mathbf{E}}^{t}\right)=\mathbf{p}_{(-)}^{\prime t}(\omega)-\mathbf{p}_{(+)}^{\prime t}(\omega),
$$

where $\mathbf{p}_{( \pm)}^{\prime t}(z)$ has zeroes and singularities for $z \in \Omega^{ \pm}$and they are defined analogously to $(5.9)$. Thus, we have

$$
\mathbf{p}_{(-)}^{\prime t}(\omega)-\mathbf{p}_{(+)}^{\prime t}(\omega)=\mathbf{p}_{(-)}^{t}(\omega)-\mathbf{p}_{(+)}^{t}(\omega)+\frac{1}{2} \check{\mathbb{G}}_{(-)}^{-1}(\omega) \breve{\mathbf{I}}_{-}^{(n)}\left(\omega, \breve{\mathbf{E}}^{t}\right)
$$

Observe that the quantity

$$
\mathbf{J}^{\prime}(\omega)=\mathbf{p}_{(+)}^{t}(\omega)-\mathbf{p}_{(+)}^{\prime t}(\omega)=\mathbf{p}_{(-)}^{t}(\omega)-{\mathbf{p}^{\prime}}_{(-)}^{t}(\omega)+\frac{1}{2} \check{\mathbb{G}}_{(-)}^{-1}(\omega) \breve{\mathbf{I}}_{-}^{(n)}\left(\omega, \breve{\mathbf{E}}^{t}\right)
$$

is analytic on $\Omega^{-}$by virtue of the first relation of $(5.18)$, and it is analytic on $\Omega^{+}$by virtue of the rightmost relation of (5.18). So $\mathbf{J}^{\prime}(\omega)=0$ and

$$
\mathbf{p}_{(+)}^{t}(\omega)=\mathbf{p}_{(+)}^{\prime t}(\omega) \text {. }
$$

In particular, when it is possible to use $(5.17)_{2}$ it follows that

$$
\frac{1}{2} \check{\mathbb{G}}_{(-)}^{-1}(\omega) \breve{\mathbf{I}}_{F}^{(R)}\left(\omega, \breve{\mathbf{E}}^{t}\right)=i \check{\mathbb{G}}_{(-)}^{-1}(\omega) \dot{\mathbb{G}}_{s}(\omega) \overline{\breve{\mathbf{E}}_{+}^{t}(\omega)}=-i \omega \check{\mathbb{G}}_{(+)}(\omega) \overline{\breve{\mathbf{E}}_{+}^{t}(\omega)},
$$

so that

$$
\mathbf{p}_{(+)}^{t}(\omega)=\mathbf{p}_{(+)}^{\prime t}(\omega)=\lim _{z \rightarrow \omega^{-}} \frac{1}{2 \pi i} \int_{-\infty}^{\infty} \frac{i \omega^{\prime} \check{\mathbb{G}}_{(+)}\left(\omega^{\prime}\right) \overline{\breve{\mathbf{E}}_{+}^{t}\left(\omega^{\prime}\right)}}{z-\omega^{\prime}} d \omega^{\prime}
$$


The quantity $\breve{\mathbf{E}}_{+}^{t}$ can be written in terms of the Fourier transform of $\mathbf{E}^{t}$ as

$$
\breve{\mathbf{E}}_{+}^{t}(\omega)=\mathbf{E}_{+}^{t}(\omega)-\frac{1}{i \omega^{+}} \mathbf{E}(t)
$$

so that

$$
\overline{\mathbf{p}_{(+)}^{t}(\omega)}=\lim _{z \rightarrow \omega^{+}} \frac{1}{2 \pi i} \int_{-\infty}^{\infty} \frac{\check{\mathbb{G}}_{(-)}\left(\omega^{\prime}\right)\left[\mathbf{E}(t)+i \omega^{\prime} \mathbf{E}_{+}^{t}\left(\omega^{\prime}\right)\right]}{\omega^{\prime}-z} d \omega^{\prime}
$$

Since

$$
\check{\mathbb{G}}_{(-)}(\omega)=\lim _{\alpha \rightarrow 0^{+}} \frac{1}{2 \pi i} \int_{-\infty}^{\infty} \frac{\check{\mathbb{G}}_{(-)}\left(\omega^{\prime}\right)}{\omega^{\prime}-(\omega+i \alpha)} d \omega^{\prime}
$$

we have

$$
\overline{\mathbf{p}_{(+)}^{t}(\omega)}=\check{\mathbb{G}}_{(-)}(\omega) \mathbf{E}(t)+i \mathbf{q}_{(-)}^{t}(\omega)
$$

where

$$
\mathbf{q}_{( \pm)}^{t}(\omega)=\lim _{\alpha \rightarrow 0^{\mp}} \frac{1}{2 \pi i} \int_{-\infty}^{\infty} \frac{\check{\mathbb{G}}_{(-)}\left(\omega^{\prime}\right) \omega^{\prime} \mathbf{E}_{+}^{t}\left(\omega^{\prime}\right)}{\omega^{\prime}-(\omega+i \alpha)} d \omega^{\prime} .
$$

It is worth noting that $\mathbf{q}_{( \pm)}^{t}(\omega)$ are the same quantity defined in [9] as $\mathbf{q}_{ \pm}^{t}(\omega)$, since $\mathbb{H}(\omega)=-\omega \dot{\mathbb{G}}_{s}(\omega)=\omega^{2} \check{\mathbb{G}}_{c}(\omega)$ and hence $\omega \check{\mathbb{G}}_{( \pm)}(\omega)$ correspond to $\mathbb{H}_{ \pm}(\omega)$ of the factorization of $\mathbb{H}$ (see (5.11) of [9]). Substitution of (5.22) into (5.13) yields

$$
\begin{aligned}
\psi_{m}(\sigma(t))= & \frac{1}{2} \mathbb{G}_{\infty} \mathbf{E}(t) \cdot \mathbf{E}(t)+\frac{1}{2 \pi} \int_{-\infty}^{\infty}\left|\check{\mathbb{G}}_{(-)}(\omega) \mathbf{E}(t)+i \mathbf{q}_{(-)}^{t}(\omega)\right|^{2} d \omega \\
= & \frac{1}{2} \mathbb{G}_{0} \mathbf{E}(t) \cdot \mathbf{E}(t)+\frac{1}{\pi} \Re \int_{-\infty}^{\infty} i \check{\mathbb{G}}_{(+)}(\omega) \mathbf{q}_{(-)}^{t}(\omega) d \omega \cdot \mathbf{E}(t) \\
& +\frac{1}{2 \pi} \int_{-\infty}^{\infty}\left|\mathbf{q}_{(-)}^{t}(\omega)\right|^{2} d \omega .
\end{aligned}
$$

On the other hand, observe that the convolutive part of the stress may be written in terms of such quantities. In fact,

$$
\begin{array}{r}
\int_{0}^{\infty} \dot{\mathbb{G}}(s) \mathbf{E}^{t}(s) d s=\frac{1}{\pi} \int_{-\infty}^{\infty} i \dot{\mathbb{G}}_{s}(\omega) \mathbf{E}_{+}^{t}(\omega) d \omega=-\frac{1}{\pi} \int_{-\infty}^{\infty} \check{\mathbb{G}}_{c}(\omega)\left(i \omega \mathbf{E}_{+}^{t}(\omega)\right) d \omega \\
=-\frac{1}{\pi} \int_{-\infty}^{\infty} \check{\mathbb{G}}_{(+)}(\omega)\left(\mathbf{q}_{(+)}^{t}(\omega)-\mathbf{q}_{(-)}^{t}(\omega)\right) d \omega
\end{array}
$$

Moreover, since both $\check{\mathbb{G}}_{(+)}(z)$ and $\mathbf{q}_{(+)}^{t}(z)$ are analytic on $\Omega^{-}$we have

$$
\begin{aligned}
& \int_{-\infty}^{\infty} \check{\mathbb{G}}_{(+)}(\omega) \mathbf{q}_{(+)}^{t}(\omega) d \omega=0, \\
& \int_{0}^{\infty} \dot{\mathbb{G}}(s) \mathbf{E}^{t}(s) d s=\frac{i}{\pi} \int_{-\infty}^{\infty} \check{\mathbb{G}}_{(+)}(\omega) \mathbf{q}_{(-)}^{t}(\omega) d \omega .
\end{aligned}
$$

Since

$$
\Re \int_{-\infty}^{\infty} i \check{\mathbb{G}}_{(+)}(\omega) \mathbf{q}_{(-)}^{t}(\omega) d \omega=\int_{-\infty}^{\infty} i \check{\mathbb{G}}_{(+)}(\omega) \mathbf{q}_{(-)}^{t}(\omega) d \omega
$$


substitution of (5.25) into (5.24) and the position

$$
S(t)=\frac{1}{2} \mathbb{G}_{0} \mathbf{E}(t) \cdot \mathbf{E}(t)+\int_{0}^{\infty} \dot{\mathbb{G}}(s) \mathbf{E}^{t}(s) d s \cdot \mathbf{E}(t)=\mathbf{T}(t) \cdot \mathbf{E}(t)-\frac{1}{2} \mathbb{G}_{0} \mathbf{E}(t) \cdot \mathbf{E}(t)
$$

leads to

$$
\psi_{m}(t)=S(t)+\frac{1}{2 \pi} \int_{-\infty}^{\infty}\left|\mathbf{q}_{(-)}^{t}(\omega)\right|^{2} d \omega
$$

that is, the expression for the minimum free energy found in [9]. As a consequence, all the properties shown in [9] for (5.26) are satisfied by (5.13) too. In particular, it satisfies the definition of the free energy stated by Graffi and the one stated by Coleman and Owen.

However, the method used here to find expression (5.13) will be useful in the next section to show that the domain of definition of the minimum free energy is the whole space of the admissible states defined in Sec. 3.

6. The space of definition of the minimum free energy. The minimum free energy $\psi_{m}$ is a function of the state $\sigma \in \Sigma_{w}$. Moreover, since $\mathbf{p}_{(+)}^{t}$ may be thought as an element of $\left(\Gamma_{w} / \Gamma_{w 0}\right),\left(\mathbf{E}(t), \mathbf{p}_{(+)}^{t}\right)$ is a direct representation of the state $\sigma(t) \in \Sigma_{w}=$ $\operatorname{Sym} \times\left(\Gamma_{w} / \Gamma_{w 0}\right)$.

This can be proved by recalling $(3.19)_{2}$ and by showing that $\breve{\mathbf{I}}\left(\tau, \breve{\mathbf{E}}^{t}\right)=0, \forall \tau>0$ if and only if $\mathbf{p}_{(+)}^{t}(\omega)=0, \forall \omega \in \mathcal{R}$. To this aim, observe that (5.5) can be rewritten as

$$
\breve{\mathbf{I}}\left(\tau, \breve{\mathbf{E}}^{t}\right)=-\int_{\tau}^{\infty} \dot{\mathbb{G}}(s) \breve{\mathbf{E}}^{t}(s-\tau) d s, \quad \tau \geq 0 .
$$

A causal extension of $\breve{\mathbf{E}}^{t}$ and an odd extension of $\dot{\mathbb{G}}$ provide the following representation of $\breve{\mathbf{I}}$ in the frequency domain:

$$
\breve{\mathbf{I}}\left(\tau, \breve{\mathbf{E}}^{t}\right)=\frac{1}{\pi} \int_{-\infty}^{\infty} i \dot{\mathbb{G}}_{s}(\omega) \overline{\breve{\mathbf{E}}_{+}^{t}(\omega)} e^{i \omega \tau} d \omega=-\frac{1}{\pi} \int_{-\infty}^{\infty} i \omega \check{\mathbb{G}}_{c}(\omega) \overline{\breve{\mathbf{E}}_{+}^{t}(\omega)} e^{i \omega \tau} d \omega, \quad \tau \geq 0 .
$$

Moreover, representation (5.20) ensures that the Plemelj formula for $i \omega \check{G}_{(+)}(\omega) \overline{\breve{\mathbf{E}}_{+}^{t}(\omega)}$ may be given by

$$
i \omega \check{\mathbb{G}}_{(+)}(\omega) \overline{\breve{\mathbf{E}}_{+}^{t}(\omega)}=\mathbf{p}_{(+)}^{t}(\omega)-\mathbf{p}_{(-)}^{\prime \prime}(\omega),
$$

where $\mathbf{p}_{(-)}^{\prime \prime}(\omega)$ is a suitable function analytic on $\Omega^{+}$. Then

$$
\begin{aligned}
\breve{\mathbf{I}}\left(\tau, \breve{\mathbf{E}}^{t}\right) & =-\frac{1}{\pi} \int_{-\infty}^{\infty} \check{\mathbb{G}}_{(-)}(\omega)\left(\mathbf{p}_{(+)}^{t}(\omega)-\mathbf{p}_{(-)}^{\prime \prime}(\omega)\right) e^{i \omega \tau} d \omega \\
& =-\frac{1}{\pi} \int_{-\infty}^{\infty} \check{\mathbb{G}}_{(-)}(\omega) \mathbf{p}_{(+)}^{t}(\omega) e^{i \omega \tau} d \omega ;, \quad \tau \geq 0,
\end{aligned}
$$

the second equality holding since $\check{G}_{(-)}(\omega), \mathbf{p}_{(-)}^{\prime t}(\omega)$, and $e^{i \omega \tau}$ are analytic in the halfplane $\Omega^{+}$. Consider now the following function:

$$
\breve{\mathbf{I}}^{t}(\tau)=-\frac{1}{\pi} \int_{-\infty}^{\infty} \check{\mathbb{G}}_{(-)}(\omega) \mathbf{p}_{(+)}^{t}(\omega) e^{i \omega \tau} d \omega ;, \quad \tau \in \mathcal{R} .
$$


This characterization of $\breve{\mathbf{I}}^{t}$ allows us to prove the following theorem.

THEOREM 6.1. For every viscoelastic material with a symmetric relaxation function, a given strain history $\breve{\mathbf{E}}^{t}$ is equivalent to the zero history $\mathbf{0}^{\dagger}$ in the sense of $(3.19)_{2}$ if and only if the quantity $\mathbf{p}_{(+)}^{t}$, related to $\breve{\mathbf{E}}^{t}$ by $(6.1)$, is such that

$$
\mathbf{p}_{(+)}^{t}(\omega)=0, \quad \forall \omega \in \mathcal{R} .
$$

Proof. Using representation (6.2), the theorem in effect states that

$$
\breve{\mathbf{I}}^{t}(\tau)=0 \quad \forall \tau \geq 0 \quad \Longleftrightarrow \quad \mathbf{p}_{(+)}^{t}(\omega)=0 \quad \forall \omega \in \mathcal{R}
$$

The statement relating to the left arrow of (6.4) follows trivially from (6.2). In order to prove the statement relating to the right arrow, we can consider (6.2) as the inverted Fourier transform of

$$
\mathbf{f}^{t}(\omega)=2 \check{\mathbb{G}}_{(-)}(\omega) \mathbf{p}_{(+)}^{t}(\omega)=-\int_{-\infty}^{\infty} \breve{\mathbf{I}}^{t}(\tau) e^{-i \omega \tau} d \tau .
$$

If $\breve{\mathbf{I}}^{t}(\tau)=0 \forall \tau \geq 0$, it follows from (6.5) that $\mathbf{f}^{t}$ is analytic in $\Omega^{+}$. The zeros of $\breve{\mathbb{G}}_{(-)}$ cannot cancel singularities of $\mathbf{p}_{(+)}^{t}$ since all such zeros are by construction in $\Omega^{-}$. Thus $\mathbf{p}_{(+)}^{t}$ must be analytic in $\Omega^{+}$and therefore in $\Omega$. Since it goes to zero at infinity, by Liouville's theorem, it must vanish and (6.3) is proved.

Theorem 6.1 ensures that $\left(\mathbf{E}(t), \mathbf{p}_{(+)}^{t}\right)$ is a direct representation of the state $\sigma(t) \in \Sigma_{w}$ and hence $\hat{\psi}_{m}\left(\mathbf{E}(t), \mathbf{p}_{(+)}^{t}\right)=\psi_{m}(\sigma(t))$ is a function of the state. More precisely, we should have to say that $\left(\mathbf{E}(t), \mathbf{p}_{(+)}^{t}\right)$ describes a state $\sigma(t) \in \mathcal{S}_{m} \subseteq \Sigma_{w}$ where

$$
\mathcal{S}_{m}:=\left\{\sigma \in \Sigma_{w}: \psi_{m}(\sigma)<\infty\right\} .
$$

Actually, any free energy is defined in a proper subset of $\Sigma_{w}$, namely

$$
\mathcal{S}:=\left\{\sigma \in \Sigma_{w}: \psi(\sigma)<\infty\right\} \subseteq \Sigma_{w}
$$

where $\psi$ is any free energy such that $\psi=0$ when evaluated at the zero history $\left(\mathbf{0}, \mathbf{0}^{\dagger}\right)$. Moreover, since $0 \leq \psi_{m}(\sigma) \leq \psi(\sigma)$, it follows that $\mathcal{S} \subseteq \mathcal{S}_{m} \subseteq \Sigma_{w}$.

Moreover, we can prove the following important result that ensures that every state of $\Sigma_{w}$ has a bounded minimum free energy.

Theorem 6.2. The space of definition of the minimum free energy is the whole state space, viz. $\mathcal{S}_{m}=\Sigma_{w}$.

Proof. Observe that a crucial equation in determining the expression of the mnimum free energy is (5.4), viz.

$$
\int_{0}^{\infty} \check{\mathbb{G}}(|\tau-s|) \dot{\mathbf{E}}^{(m)}(s) d s=\breve{\mathbf{I}}\left(\tau, \breve{\mathbf{E}}^{t}\right), \quad \tau \in \mathcal{R}^{+} .
$$

Substitution of (6.8) in (5.2) and (5.3) yields

$$
\begin{aligned}
W_{R}(\sigma) & =\frac{1}{2} \mathbb{G}_{\infty} \mathbf{E}(t) \cdot \mathbf{E}(t)+\frac{1}{2} \int_{0}^{\infty} \int_{0}^{\infty} \check{\mathbb{G}}(|\tau-s|) \dot{\mathbf{E}}^{(m)}(s) \cdot \dot{\mathbf{E}}^{(m)}(\tau) d s d \tau \\
& =\frac{1}{2} \mathbb{G}_{\infty} \mathbf{E}(t) \cdot \mathbf{E}(t)+\frac{1}{2 \pi} \int_{-\infty}^{\infty} \check{\mathbb{G}}_{c}(\omega) \dot{\mathbf{E}}_{+}^{(m)}(\omega) \cdot \overline{\dot{\mathbf{E}}_{+}^{(m)}(\omega)} d \omega .
\end{aligned}
$$


Therefore, $W_{R}(\sigma)$ induces a natural norm and a natural functional space for the processes $P \in \Pi^{0}$, namely,

$$
\check{\mathcal{H}}_{G}^{0}\left(\mathcal{R}^{+}, \text {Sym }\right):=\left\{\boldsymbol{\varphi} \in \check{\mathcal{H}}_{G}\left(\mathcal{R}^{+}, S y m\right): \int_{0}^{\infty} \boldsymbol{\varphi}(\tau) d \tau=\mathbf{0}\right\} .
$$

Moreover, it is worth noting that (6.9) and Theorems 4.2 and 4.4 ensure that $\check{\mathcal{H}}_{G}^{0}\left(\mathcal{R}^{+}\right.$, Sym) is dense in $\mathcal{H}_{G}\left(\mathcal{R}^{+}\right.$, Sym $)$with respect to the norm $\|\cdot\|_{G}$. In fact, considering the fixed value of Theorem 4.4, equations (4.30) and (4.31) and Theorem 4.2 imply

$$
\sup \{-W(\sigma, P), P \in \Pi\}=\sup \left\{\frac{1}{2} \mathbb{G}_{\infty} \mathbf{E}(t) \cdot \mathbf{E}(t)-W(\sigma, P), P \in \Pi^{0}\right\} .
$$

Equations (6.9) and (6.11), jointly with definitions (4.1) and (3.12), ensure that $\check{\mathcal{H}}_{G}^{0}\left(\mathcal{R}^{+}\right.$, Sym) and $\check{\mathcal{H}}_{G}\left(\mathcal{R}^{+}\right.$, Sym $)$admit the same completion with respect to the norm $\|\cdot\|_{G}$.

As a consequence, every state $\sigma(t)$, whose representative strain history $\left(\mathbf{E}(t) \breve{\mathbf{E}}^{t}\right)$ is such that $\mathbf{E}(t) \in S y m$ and $\breve{\mathbf{I}}\left(\cdot, \breve{\mathbf{E}}^{t}\right) \in \mathcal{H}_{G}^{\prime}\left(\mathcal{R}^{+}, S y m\right)$, belongs to $\mathcal{S}_{m}$, so that $\Sigma_{w} \subseteq \mathcal{S}_{m}$. Since definition (6.6) implies $\mathcal{S}_{m} \subseteq \Sigma_{w}$, the thesis is proved.

We conclude the section by characterizing the states of $\Sigma_{w}$ and, in particular, analyzing the type of past strain histories $\breve{\mathbf{E}}^{t}$ belonging to $\Gamma_{w} / \Gamma_{w 0}$. Remember that an element of $\Gamma_{w} / \Gamma_{w 0}$ can be directly represented by $\mathbf{p}_{(+)}^{t}$ as a consequence of Theorem 6.1 .

Proposition 6.3. Given a past strain history $\breve{\mathbf{E}}^{t}$, then $\breve{\mathbf{I}}\left(t, \breve{\mathbf{E}}^{t}\right) \in \mathcal{H}_{G}^{\prime}\left(\mathcal{R}^{+}, S y m\right)$ if and only if $\mathbf{p}_{(+)}^{t} \in L^{2}(\mathcal{R}, \operatorname{Sym}(\Omega))$.

Proof. For every $\varphi \in \mathcal{H}_{G}\left(\mathcal{R}^{+}\right.$, Sym $)$we have

$$
\int_{-\infty}^{\infty} \check{\mathbb{G}}_{c}(\omega) \boldsymbol{\varphi}_{+}(\omega) \cdot \overline{\boldsymbol{\varphi}_{+}(\omega)} d \omega=\int_{-\infty}^{\infty}\left|\check{\mathbb{G}}_{(+)}(\omega) \boldsymbol{\varphi}_{+}(\omega)\right|^{2} d \omega .
$$

Consider now $\varphi \in \mathcal{H}_{G}\left(\mathcal{R}^{+}, S y m\right)$ and the duality product

$$
\begin{aligned}
\int_{0}^{\infty} \breve{\mathbf{I}}\left(t, \mathbf{E}^{t}\right) \cdot \boldsymbol{\varphi}(t) d t & =\frac{1}{2 \pi} \int_{-\infty}^{\infty} \breve{\mathbf{I}}_{+}^{t}(\omega) \cdot \overline{\boldsymbol{\varphi}_{+}(\omega)} d \omega \\
& =\frac{1}{2 \pi} \int_{-\infty}^{\infty} \check{\mathbb{G}}_{(-)}^{-1}(\omega) \breve{\mathbf{I}}_{+}^{t}(\omega) \cdot\left(\check{\mathbb{G}}_{(-)}^{\top}(\omega) \overline{\boldsymbol{\varphi}_{+}(\omega)}\right) d \omega \\
& =\frac{1}{2 \pi} \int_{-\infty}^{\infty} \check{\mathbb{G}}_{(-)}^{-1}(\omega) \breve{\mathbf{I}}_{+}^{t}(\omega) \cdot\left(\check{\mathbb{G}}_{(+)}(\omega) \boldsymbol{\varphi}_{+}(\omega)\right) d \omega
\end{aligned}
$$

The Plemelj formulae (5.8) and the fact that

$$
\int_{-\infty}^{\infty} \mathbf{p}_{(-)}^{t}(\omega) \cdot\left(\check{\widetilde{G}}_{(+)}(\omega) \varphi_{+}(\omega)\right) d \omega=0
$$

lead to

$$
\begin{aligned}
\int_{0}^{\infty} \breve{\mathbf{I}}\left(t, \mathbf{E}^{t}\right) \cdot \boldsymbol{\varphi}(t) d t & =\frac{1}{2 \pi} \int_{-\infty}^{\infty} \mathbf{p}_{(+)}^{t}(\omega) \cdot\left(\overline{\breve{G}_{(+)}(\omega) \varphi_{+}(\omega)}\right) d \omega \\
& \leq \frac{1}{4 \pi} \int_{-\infty}^{\infty}\left|\mathbf{p}_{(+)}^{t}(\omega)\right|^{2} d \omega+\frac{1}{4 \pi} \int_{-\infty}^{\infty}\left|\check{\mathbb{G}}_{(+)}(\omega) \varphi_{+}(\omega)\right|^{2} d \omega
\end{aligned}
$$

Therefore, if $\mathbf{p}_{(+)}^{t} \in L^{2}(\mathcal{R}, \operatorname{Sym}(\omega))$, then $\breve{\mathbf{I}}\left(\cdot, \mathbf{E}^{t}\right) \in \mathcal{H}_{G}^{\prime}\left(\mathcal{R}^{+}, S y m\right)$. 
On the other hand, if $\breve{\mathbf{I}}\left(\cdot, \mathbf{E}^{t}\right) \in \mathcal{H}_{G}^{\prime}\left(\mathcal{R}^{+}\right.$, Sym $)$, Theorem 6.2 ensures that the maximum recoverable work is finite and the optimal process $\dot{\mathbf{E}}^{(m)} \in \mathcal{H}_{G}\left(\mathcal{R}^{+}\right.$, Sym). Thus we have

$$
0 \leq \int_{-\infty}^{\infty} \check{\mathbb{G}}_{c}(\omega) \dot{\mathbf{E}}_{+}^{(m)}(\omega) \cdot \overline{\dot{\mathbf{E}}_{+}^{(m)}(\omega)} d \omega=\frac{1}{2} \mathbb{G}_{\infty} \mathbf{E}(t) \cdot \mathbf{E}(t)-W_{R}(\sigma)<\infty .
$$

Substitution of (5.11) in (6.12), jointly with (5.7) and the property $\check{\mathbb{G}}_{( \pm)}=\check{\mathbb{G}}_{(\mp)}^{*}$, yield

$$
\int_{-\infty}^{\infty}\left|\mathbf{p}_{(+)}^{t}(\omega)\right|^{2} d \omega<\infty .
$$

Therefore, $\breve{\mathbf{I}}\left(\cdot, \mathbf{E}^{t}\right) \in \mathcal{H}_{G}^{\prime}\left(\mathcal{R}^{+}\right.$, Sym $)$implies $\mathbf{p}_{(+)}^{t} \in L^{2}(\mathcal{R}, \operatorname{Sym}(\omega))$.

7. Recoverable work and thermodynamics in viscoelasticity. The maximum recoverable work and its properties may be taken as the basis for the thermodynamics of a viscoelastic material under isothermal conditions. From this point of view, it is of interest to recall the Dissipation Principle of the Mechanical Energy in the form stated by Fabrizio, Giorgi and Morro in [11] that reads:

Dissipation PRINCIPLE. For any state $\sigma \in \Sigma_{w}$, the set of works done starting from $\sigma$ is bounded from below, viz.

$$
N(\sigma):=\inf \{W(\sigma, P): P \in \Pi\}>-\infty .
$$

In our context, we do not need to assume such a Principle. In fact, we can prove it by virtue of Theorem 6.2 and of a "Weak Dissipation Principle" that is actually a definition of a new state space, called "space of admissible states" and that reads:

Weak Dissipation Principle. There exists a suitable state space $\Sigma_{N} \subseteq \Sigma_{w}$, named as the set of admissible state, whose elements are all and only those states $\sigma \in \Sigma_{w}$ satisfying (7.1).

In other words,

$$
\Sigma_{N}:=\left\{\sigma \in \Sigma_{w}: N(\sigma)>-\infty\right\} .
$$

Observe that, comparing (7.1) with $(4.1)$, it is clear that

$$
W_{R}(\sigma)=-N(\sigma)<\infty \text {. }
$$

Thus Definition 4.1 and comparison (7.3) clarify the physical meaning of the name "admissible state" for a state $\sigma$ satisfying (7.1). In fact, if (7.1) does not hold, we might have a state that could provide infinite energy, and this is not physically admissible.

Moreover, by virtue of (5.1), it is easy to note that the spaces $\mathcal{S}_{m}$ and $\Sigma_{N}$, defined respectively by (6.6) and (7.2), coincide. As a consequence, Theorem 6.2, which ensures $\mathcal{S}_{m}=\Sigma_{w}$, proves the Dissipation Principle too.

Such a thermodynamic principle, in the form introduced in [11] or in the weaker form shown above, agrees with the general theory of dissipative dynamical systems proposed by Willems in [25]. In fact, when a viscoelastic material under isothermal conditions is considered, the general "supply rate" of [25] corresponds to the mechanical power, the general "storage function" $S$ of [25] is the free energy and the "available storage" $S$ of [25] is the maximum recoverable work (i.e., the minimum frec energy). From this point of view it is worth quoting Theorem 1 of [25] that reads as follows. 
Theorem 7.1 (Willems). The available storage (i.e., the maximum recoverable work) $W_{R}(\sigma)$ is finite for every $\sigma \in \Sigma$ if and only if the material is dissipative. Moreover, $0 \leq W_{R}(\sigma) \leq \psi(\sigma)(\forall \sigma \in \Sigma)$ and $W_{R}(\sigma)$ is itself a possible storage function (free energy).

This theorem points out how Theorem 4.2 is crucial. In other words, Theorem 7.1 asserts that assuming the dissipative property of the material is equivalent to state that the domain of definition of the minimum free energy coincides with the whole state space.

Let us recall how (7.1) leads to the Clausius-Duhem inequality. Remember that $W_{R}$ satisfies the following property.

Proposition 7.2. The maximum recoverable work $W_{R}(\sigma)$ is nonnegative for every $\sigma \in$ $\Sigma_{m}$.

The proof is an immediate consequence of (4.1), recalling that at every state $\sigma$ it is possible to apply the null process yielding a null work.

We recall the following assumption that is quite natural at least for simple materials and, in particular, for viscoelastic materials (see [11]).

Assumption 7.3. There exists a "zero state" $\sigma_{0}$, such that $W_{R}\left(\sigma_{0}\right)=0$.

Actually, as pointed out in [11], for viscoelastic materials, $\sigma_{0}$ is the state related to the identically vanishing strain $\left(\mathbf{0}, \mathbf{0}^{\dagger}\right)$.

The fundamental principles of thermodynamics follow from the Dissipation Principle and Assumption 7.3. In fact, from (7.1) and (4.1) it follows that for each fixed pair $\sigma \in \Sigma_{w}, P_{1} \in \Pi$, we have $\Pi_{1}:=\left\{P=P^{\prime} * P_{1}, P^{\prime} \in \Pi\right\} \subset \Pi$ so that

$$
\begin{aligned}
W_{R}(\sigma) & \geq \sup \left\{-W\left(\sigma, P^{\prime} * P_{1}\right), P^{\prime} \in \Pi\right\} \\
& =-W\left(\sigma, P_{1}\right)+\sup \left\{-W\left(P_{1} \sigma, P^{\prime}\right), P^{\prime} \in \Pi\right\}=-W\left(\sigma, P_{1}\right)+W_{R}(P \sigma) .
\end{aligned}
$$

Therefore, for any $\sigma_{1}, \sigma_{2} \in \Sigma_{w}$ and $P \in \Pi$ such that $P \sigma_{1}=\sigma_{2}$, we have

$$
W_{R}\left(\sigma_{2}\right)-W_{R}\left(\sigma_{1}\right) \leq W\left(\sigma_{1}, P\right) .
$$

As a consequence, for every cycle, that is, a pair $\sigma \in \Sigma_{w}$ and $P \in \Pi$ such that $P \sigma=\sigma$, (7.4) implies

$$
W(\sigma, P) \geq 0
$$

that is, the Second Principle of Thermodynamics in the formulation of Clausius. Instead, Assumption 7.3 implies the Dissipation Principle of Gurtin and Herrera [22]. In fact, from Assumption 7.3 and (4.1) it follows that

$$
W\left(\sigma_{0}, P\right) \geq 0 \quad \forall P \in \Pi,
$$

as stated by the above-mentioned Principle.

Inequality (7.5) implies that there exists a function of state $\psi(\sigma)$, called free energy, that is a lower potential such that

$$
\psi\left(\sigma_{2}\right)-\psi\left(\sigma_{1}\right) \leq W\left(\sigma_{1}, P\right),
$$


that is, the integrated Clausius -Duhem inequality for isothermal processes. ${ }^{2}$ Comparing (7.4) with (7.6), it is clear that $W_{R}$ is a free energy.

We conclude the section by discussing the physical meaning of the quantity

$$
D\left(\sigma_{1}, P\right):=W\left(\sigma_{1}, P\right)-\psi\left(\sigma_{2}\right)+\psi\left(\sigma_{1}\right) \geq 0 .
$$

In fact, $D$ is usually called as the dissipation of the material, so that a process $P$, acting on $\sigma$, should be reversible (i.e., nondissipative) if and only if $D(\sigma, P)=0$ and a material should be nondissipative if and only if $D(\sigma, P)=0$ for every $\sigma \in \Sigma$ and $P \in \Pi$.

However, this way of relating the dissipation to the Clausius-Duhem inequality (7.6) fails when memory effects occur, as pointed out in [12]. This is substantially due to the nonuniqueness of the free energy as a functional satisfying (7.6), whereas the dissipation should be an observable uniquely determined. Moreover, we can find a counterexample of a suitable free energy satisfying (7.6) and (7.7) as an equality even if the material with memory is dissipative. This is the case for the maximum free energy defined in [11].

Even in this situation the maximum recoverable work shows an interesting property. In fact, inequality (7.4), unlike (7.6), is uniquely fixed and it represents the dissipation of the material by virtue of the definition of the maximum recoverable work as the amount of available energy. In fact, (7.4) can be interpreted as follows: the sum given by the energy provided to the material by means of the work $W\left(\sigma_{1}, P\right)$ added to the available energy at the state $\sigma_{1}$ is not less than the available energy at the state $P \sigma_{1}=\sigma_{2}$. The difference is the part of $W\left(\sigma_{1}, P\right)$ that cannot be utilized anymore, that is, the dissipated energy. Therefore, denoting by $D_{i}$ the function defined in (7.7) when $\psi_{i}$ is the free energy involved, and calling $\psi_{m}$ the free energy given by the maximum recoverable work, viz.

$$
\psi_{m}(\sigma)=W_{R}(\sigma),
$$

in view of (7.4), (7.7) and (7.8) the quantity

$$
D_{m}\left(\sigma_{1}, P\right):=W\left(\sigma_{1}, P\right)-\psi_{m}\left(\sigma_{2}\right)+\psi_{m}\left(\sigma_{1}\right) \geq 0
$$

represents the dissipation of the material in the sense outlined above.

Thus, when memory effects occur, the Clausius-Duhem inequality is still related to the dissipation of the material provided the involved free energy is the one given by the maximum recoverable work.

Appendix: Factorization of the integral kernel. The solution of the WienerHopf equation (5.4) relies crucially on the factorization of the integral kernel. The aim of this appendix is to recall the results of Gohberg and Kreın [16] and Deseri, Gentili and Golden [9], which ensure such a factorization. The results in [16] apply to Hermitian matrices of arbitrary finite dimension but we state them for the case of $6 \times 6$ matrices; moreover, as recalled in Sec. 2, they can be easily extended to tensors belonging to $\operatorname{Lin}(\operatorname{Sym}(\Omega))$. The result in [9] shows sufficient conditions for the factorization of the integral kernel $\check{\mathbb{G}}$.

\footnotetext{
${ }^{2}$ However, inequality (7.6) allows many functionals to be a free energy, especially for a material with memory, and they do not differ just for an additive constant.
} 
Definition A.1. A nonsingular continuous function $\mathbb{K}: \mathcal{R} \rightarrow \operatorname{Lin}(\operatorname{Sym}(\Omega))$ has a left [right] factorization if. for every $\omega \in \mathcal{R}$, it can be represented in the form

$$
\mathbb{K}(\omega)=\mathbb{K}_{(+)}(\omega) \mathbb{K}_{(-)}(\omega) \quad\left[\mathbb{K}(\omega)=\mathbb{K}_{(-)}(\omega) \mathbb{K}_{(+)}(\omega)\right],
$$

where the matrix functions $\mathbb{K}_{( \pm)}$admit analytic continuations, holomorphic in the interior and continuous up to the boundary of the corresponding complex half planes $\Omega^{ \pm}$, and are such that

$$
\operatorname{det} \mathbb{K}_{( \pm)}(\zeta) \neq 0, \quad \zeta \in \Omega^{ \pm}
$$

Definition A.2. A nonsingular continuous function $\mathbb{K}: \mathcal{R} \rightarrow \operatorname{Lin}(\operatorname{Sym}(\Omega))$ belongs to $\Re_{6 \times 6},\left[\Re_{6 \times 6}^{+}\right],\left[\left[\Re_{6 \times 6}^{-}\right]\right]$if there exists a constant matrix $\mathbb{C}_{0}$ and a matrix function $\mathbb{F}(t)$ such that

$$
\begin{gathered}
\mathbb{K}(\omega)=\mathbb{C}_{0}+\int_{-\infty}^{\infty} \mathbb{F}(t) e^{i \omega t} d t, \quad \forall \omega \in \mathcal{R}, \\
{\left[\mathbb{K}(\omega)=\mathbb{C}_{0}+\int_{0}^{\infty} \mathbb{F}(t) e^{i \omega t} d t\right], \quad\left[\left[\mathbb{K}(\omega)=\mathbb{C}_{0}+\int_{-\infty}^{0} \mathbb{F}(t) e^{i \omega t} d t\right]\right] .}
\end{gathered}
$$

Note that if $\mathbb{K} \in \Re_{6 \times 6}^{ \pm}$, it has the analytic properties ascribed to $\mathbb{K}_{( \pm)}$above. The main result we use is Theorem 8.2 of [16], that can be stated in our context as follows:

Theorem A.3 (Gohberg Krein). In order that the nonsingular (Hermitian) matrix function $\mathbb{K} \in \Re_{6 \times 6}$ possess a representation of the form

$$
\mathbb{K}(\omega)=\mathbb{K}_{(+)}(\omega) \mathbb{K}_{(+)}^{*}(\omega),
$$

in which the matrix function $\mathbb{K}_{(+)} \in \Re_{6 \times 6}^{+}$and satisfies $\operatorname{det} \mathbb{K}_{(+)}(\zeta) \neq 0$ for $\zeta \in \Omega^{+}$, it is necessary and sufficient that $\mathbb{K}(\omega)$ be positive definite for every $-\infty \leq \omega \leq \infty$.

Comparison of (A.5) with the first relation of (A.1) yields

$$
\mathbb{K}_{(-)}(\omega)=\mathbb{K}_{(+)}^{*}(\omega) \text {. }
$$

Recalling that any fourth-order symmetric tensor maps into a $6 \times 6$ matrix under the isomorphism discussed at the beginning of Section 2, let us consider for each given $\omega \in \mathcal{R}$ the fourth-order tensor $\check{\mathbb{G}}_{c}(\omega) \in \operatorname{Lin}($ Sym) defined by (2.6). By virtue of $(2.7)$ and the assumption that $\check{\mathbb{G}}(t)$ is symmetric, it follows that $\check{\mathbb{G}}_{c}(\omega)$ is a real-valued, symmetric, positive definite tensor. Moreover, since

$$
\lim _{\omega \rightarrow \infty} \omega^{2} \check{\mathbb{G}}_{c}(\omega)=-\lim _{\omega \rightarrow \infty} \omega \dot{\mathbb{G}}_{s}(\omega)=-\dot{\mathbb{G}}(0),
$$

it follows from (2.9) that the tensor

$$
\mathbb{K}(\omega):=\left(1+\omega^{2}\right) \check{\mathbb{G}}_{c}(\omega)
$$

is symmetric and positive definite for every $-\infty \leq \omega \leq \infty$.

The result in $[9]$ ensures that $\mathbb{K} \in \Re_{6 \times 6}$, i.e., that the representation (A.3) applies to $\mathbb{K}$.

Proposition A.4 (Deseri-Gentili-Golden). If $\check{G}$ and $\ddot{\mathbb{G}}$ are integrable tensor-valued functions, and the tensor $\dot{\mathbb{G}}(0)$ is finite and nonsingular, the tensor-valued function $\mathbb{K}$, related to $\mathbb{G}$ through (A.8), belongs to $\Re_{6 \times 6}$. 
Proof. Observe that integration by parts yields

$$
\omega^{2} \check{\mathbb{G}}_{c}(\omega)=-\omega \dot{\mathbb{G}}_{s}(\omega)=-\dot{\mathbb{G}}(0)-\ddot{\mathbb{G}}_{c}(\omega),
$$

so that (A.8) becomes

$$
\mathbb{K}(\omega)=\left[-\dot{\mathbb{G}}(0)-\ddot{\mathbb{G}}_{c}(\omega)+\check{\mathbb{G}}_{c}(\omega)\right] .
$$

Consider now the tensors

$$
\mathbb{C}_{0}=-\dot{\mathbb{G}}(0), \quad \mathbb{F}(t)=\frac{1}{2}[-\ddot{\mathbb{G}}(t)+\check{\mathbb{G}}(t)], \quad t \in \mathcal{R},
$$

where $\check{\mathbb{G}}$ and $\ddot{\mathbb{G}}$ are extended on the real line as even functions, so that $\check{\mathbb{G}}_{F}=2 \check{\mathbb{G}}_{c}$ and $\ddot{\mathbb{G}}_{F}=2 \ddot{\mathbb{G}}_{c}$. Then, in view of $(\mathrm{A} .11),(\mathrm{A} .10)$ is equivalent to (A.3) and the assertion is proved.

Theorem A.3, Proposition A.4, and suitable hypotheses on $\ddot{\mathbb{G}}$ ensure that $\mathbb{K}(\omega)$ has a representation of the form (left factorization)

$$
\mathbb{K}(\omega)=\mathbb{K}_{(+)}(\omega) \mathbb{K}_{(-)}(\omega)
$$

with $\mathbb{K}_{(-)}(\omega)=\mathbb{K}_{(+)}^{*}(\omega), \mathbb{K}_{(+)}(\omega) \in \Re_{6 \times 6}^{+}$and

$$
\operatorname{det} \mathbb{K}_{(+)}(\zeta) \neq 0 \quad \text { for } \quad \zeta \in \Omega^{+} \text {. }
$$

Moreover, such a factorization is unique up to a post-multiplication on the right by a constant unitary tensor (see Remark on p. 253 of [16]).

As a consequence $\check{\mathbb{G}}_{c}(\omega)$ can be factorized too. In fact, we have

$$
\check{\mathbb{G}}_{c}(\omega)=\check{\mathbb{G}}_{(+)}(\omega) \check{\mathbb{G}}_{(-)}(\omega)
$$

where

$$
\check{\mathbb{G}}_{( \pm)}(\omega)=\frac{1}{1 \pm i \omega} \mathbb{K}_{( \pm)}(\omega) \text {. }
$$

Observe that, following the convention used in [17], both for $\mathbb{K}_{( \pm)}$and for $\check{\mathbb{G}}_{( \pm)}$the sign indicates the half-plane where any singularities of the tensors $\mathbb{K}_{( \pm)}(\zeta)$ and $\check{\mathbb{G}}_{( \pm)}(\zeta)$, $\zeta \in \Omega$ and any zeros in the determinant of the corresponding matrices may occur.

\section{REFERENCES}

[1] S. Breuer and E. T. Onat, On the maximum recoverable work in linear viscoelasticity, Z. Angew. Math. Phys. 15, 12-21 (1964)

[2] S. Breuer and E. T. Onat, On the determination of free energy in linear viscoelasticity, Z. Angew. Math. Phys. 15, 184-191 (1964)

[3] B. D. Coleman and D. R. Owen, A mathematical foundation for thermodynamics, Arch. Rational Mech. Anal. 54, 1-104 (1974)

[4] W. A. Day, Reversibility, recoverable work and free energy in linear viscoelasticity, Quart. J. Mech. Appl. Math. 23, 1-15 (1970)

[5] W. A. Day, Some results on the least work needed to produce a given strain in a given time in a viscoelastic material and a uniqueness theorem for dynamic viscoelasticity, Quart. J. Mech and Appl. Math. 23, 469-479 (1970)

[6] W. A. Day, The Thermodynamics of Simple Materials with Fading Memory, Springer Tracts in Natural Philosophy, vol. 22, Springer-Verlag, New York, 1972

[7] G. Del Piero and L. Deseri, On the analytic expression of the free energy in linear viscoelasticity, J. Elasticity 43, 247-278 (1996) 
[8] G. Del Piero and L. Deseri, On the concepts of state and free energy in linear viscoelasticity, Arch. Rational Mech. Anal. 138, 1-35 (1997)

[9] L. Deseri, G. Gentili, and J. M. Golden, An explicit formula for the minimum free energy in linear viscoelasticity, J. of Elasticity 54, 141-185 (1999)

[10] E. H. Dill, Simple materials with fading memory, in Continuum Physics II, A.C. Eringen ed., Academic Press, New York, 1975

[11] M. Fabrizio, C. Giorgi, and A. Morro, Free energies and dissipation properties for systems with memory, Arch. Rational Mech. Anal. 125, 341-373 (1994)

[12] M. Fabrizio, C. Giorgi, and A. Morro, Internal dissipation, relaxation property, and free energy in materials with fading memory, J. Elasticity 40, 107-122 (1995)

[13] M. Fabrizio and J. M. Golden, Maximum and minimum free energies for a linear viscoelastic material, Quart. Appl. Math., to appear

[14] M. Fabrizio and A. Morro, Viscoelastic relaxation functions compatible with thermodynamics, J. Elasticity 19, 63-75 (1988)

[15] M. Fabrizio and A. Morro, Mathematical Problems in Linear Viscoelasticity, SIAM, Philadelphia, 1992

[16] I. C. Gohberg and M. G. Krĕn, Systems of integral equations on a half-line with kernels depending on the difference of arguments, Amer. Math. Soc. Transl. Ser. 2, 14, 217-287 (1960)

[17] J. M. Golden, Free energies in the frequency domain: The scalar case, Quart. Appl. Math. 58, 127-150 (2000)

[18] D. Graffi, Sull'espressione dell'energia libera nei materiali viscoelastici lineari, Ann. di Mat. Pura e Appl. (IV), 98, 273-279 (1974)

[19] D. Graffi, Sull'espressione analitica di alcune grandezze termodinamiche nei materiali con memoria, Rend. Sem. Mat. Univ. Padova 68, 17-29 (1982)

[20] D. Graffi, Ancora sull'espressione analitica dell'energia libera nei materiali con memoria, Atti Acc. Scienze Torino 120, 111-124 (1986)

[21] D. Graffi and M. Fabrizio, Sulla nozione di stato materiali viscoelastici di tipo 'rate', Atti Accad. Naz. Lincei 83, 201-208 (1990)

[22] M. E. Gurtin and I. Herrera, On dissipation inequalities and linear viscoelasticity, Quart. Appl. Math. 23, 235-245 (1965)

[23] A. Morro and M. Vianello, Minimal and maximal free energy for materials with memory, Boll. Un. Mat. Ital. 4A, 45-55 (1990)

[24] W. Noll, A new mathematical theory of simple materials, Arch. Rational Mech. Anal. 48, 1-50 (1972)

[25] J. C. Willems, Dissipative dynamical systems. Part I: General theory, Arch. Rational Mech. Anal. 45, 321-351 (1972) 